\title{
Molecular Detection of Tick-Borne Pathogen Diversities in Ticks from Livestock and Reptiles along the Shores and Adjacent Islands of Lake Victoria and Lake Baringo, Kenya
}

\author{
David Omondi ${ }^{1,2,3}$, Daniel K. Masiga', Burtram C. Fielding ${ }^{2}$, Edward Kariuki ${ }^{4}$, \\ Yvonne Ukamaka Ajamma ${ }^{1,5}$, Micky M. Mwamuye ${ }^{1}$, Daniel O. Ouso ${ }^{1,5}$ \\ and Jandouwe Villinger ${ }^{1 *}$
}

IInternational Centre of Insect Physiology and Ecology (icipe), Nairobi, Kenya, ${ }^{2}$ University of Western Cape, Bellville, South Africa, ${ }^{3}$ Egerton University, Egerton, Kenya, ${ }^{4}$ Kenya Wildlife Service, Nairobi, Kenya, ${ }^{5}$ Jomo Kenyatta University of Agriculture and Technology, Nairobi, Kenya

OPEN ACCESS

Edited by:

Dirk Werling,

Royal Veterinary College, UK

Reviewed by:

Timothy Connelley,

University of Edinburgh, UK

Abdul Jabbar,

University of Melbourne, Australia

Ria Ghai,

Emory University, USA

*Correspondence:

Jandouwe Villinger

jandouwe@icipe.org

Specialty section:

This article was submitted to Veterinary Infectious Diseases,

a section of the journal

Frontiers in Veterinary Science

Received: 07 December 2016

Accepted: 27 April 2017

Published: 01 June 2017

Citation:

Omondi D, Masiga DK, Fielding BC,

Kariuki E, Ajamma YU,

Mwamuye MM, Ouso DO and Villinger J (2017) Molecular Detection of Tick-Borne Pathogen Diversities

in Ticks from Livestock and

Reptiles along the Shores and Adjacent Islands of Lake Victoria and Lake Baringo, Kenya.

Front. Vet. Sci. 4:73.

doi: 10.3389/fvets.2017.00073
Although diverse tick-borne pathogens (TBPs) are endemic to East Africa, with recognized impact on human and livestock health, their diversity and specific interactions with tick and vertebrate host species remain poorly understood in the region. In particular, the role of reptiles in TBP epidemiology remains unknown, despite having been implicated with TBPs of livestock among exported tortoises and lizards. Understanding TBP ecologies, and the potential role of common reptiles, is critical for the development of targeted transmission control strategies for these neglected tropical disease agents. During the wet months (April-May; October-December) of 2012-2013, we surveyed TBP diversity among 4,126 ticks parasitizing livestock and reptiles at homesteads along the shores and islands of Lake Baringo and Lake Victoria in Kenya, regions endemic to diverse neglected tick-borne diseases. After morphological identification of 13 distinct Rhipicephalus, Amblyomma, and Hyalomma tick species, ticks were pooled ( $\leq 8$ individuals) by species, host, sampling site, and collection date into 585 tick pools. By supplementing previously established molecular assays for TBP detection with high-resolution melting analysis of PCR products before sequencing, we identified high frequencies of potential disease agents of ehrlichiosis (12.48\% Ehrlichia ruminantium, 9.06\% Ehrlichia canis), anaplasmosis (6.32\% Anaplasma ovis, 14.36\% Anaplasma platys, and 3.08\% Anaplasma bovis,), and rickettsiosis (6.15\% Rickettsia africae, 2.22\% Rickettsia aeschlimannii, 4.27\% Rickettsia rhipicephali, and 4.95\% Rickettsia spp.), as well as Paracoccus sp. and apicomplexan hemoparasites (0.51\% Theileria sp., 2.56\% Hepatozoon fitzsimonsi, and $1.37 \%$ Babesia caballi) among tick pools. Notably, we identified E. ruminantium in both Amblyomma and Rhipicephalus pools of ticks sampled from livestock in both study areas as well as in Amblyomma falsomarmoreum (66.7\%) and Amblyomma nuttalli (100\%) sampled from tortoises and Amblyomma sparsum (63.6\%) sampled in both cattle and tortoises at Lake Baringo. Similarly, we identified E. canis in rhipicephaline ticks sampled from livestock and dogs in both regions and Amblyomma latum (75\%) sampled from monitor lizards at Lake Victoria. These novel tick-host-pathogen interactions have implications on the risk of disease transmission 
to humans and domestic animals and highlight the complexity of TBP ecologies, which may include reptiles as reservoir species, in sub-Saharan Africa.

Keywords: tick-borne diseases, Ehrlichia, Anaplasma, Rickettsia, Babesia, Hepatozoon, Theileria, Kenya

\section{INTRODUCTION}

Tick-borne pathogens (TBPs) are responsible for some of the most serious emerging infectious diseases facing sub-Saharan Africa (SSA) and the rest of the world today $(1,2)$. In Kenya, TBPs (including viral diseases-arboviruses) like Crimean Congo hemorrhagic fever (CCHF), Dugbe, Kupe, and Hazara, as well as hemoparasites that cause babesiosis, theileriosis, and rickettsiosis, are major impediments to livestock productivity and public health (3-6).

Baringo and Homa Bay counties of Kenya are both characterized by unique land-lake biogeographies with fluctuating fresh water levels of Lakes Baringo and Victoria, respectively. These ecological habitats form shallow lagoons with abundant aquatic and terrestrial biodiversity that include diverse vertebrate host species and disease vectors (7). The interaction of domestic animals (mainly livestock, dogs, chickens, and cats), migratory birds, humans, and wildlife has the potential to facilitate the spread of zoonotic pathogens, including TBPs. Wildlife can act as both sources and maintenance hosts for TBPs (8) that can also be transmitted to livestock and humans $(9,10)$, causing significant morbidity and mortality (11). For example, East Coast fever (ECF), caused by Theileria parva, originates from African buffalo and circulates in cattle, which often succumb to the disease $(12,13)$.

Recent studies have implicated reptiles as potential reservoirs involved in TBP transmission cycles. Ehrlichia ruminantium, considered a pathogen of ruminants responsible for heartwater disease, has been reported among Amblyomma sparsum (tortoise tick) sampled from leopard tortoises imported into the United States from Zambia (14). Furthermore, diverse Borrelia, Rickettsia, Ehrlichia, Anaplasma, and Babesia species have been identified in lizard species in Portugal (15), Australia (16), and the Netherlands (17), as well as in diverse reptiles imported into Japan (18). These studies suggest a possible role of reptiles in the epidemiology of diverse tick-borne diseases, including heartwater, which has not been investigated in African endemic settings.

Increased TBP sharing between wildlife and livestock species may result from human-induced interactions $(19,20)$. Nomadic and pastoralist lifestyles lead to direct and indirect contact that facilitate exposure and sharing of previously isolated pathogens $(21,22)$. Wildlife translocations to new habitats have also resulted in outbreaks and mortality among naive inhabitants, as was the case in cattle when T. parva-infected buffalo was translocated to the Highveld of Zimbabwe (23).

In Baringo County, the burden of tick-borne diseases remains largely unknown. The pastoralist communities that inhabit Baringo County plains keep large herds of livestock that are parasitized by an abundant diversity of vector tick species with pathogens that inflict significant economic losses on already drought impoverished populations (24). Interestingly, tick-laden free ranging tortoises that scavenge for food are common in and around homesteads where they interact with humans and livestock. Such domestic-wildlife interactions increase the likelihood of ticks and their pathogens parasitizing different vertebrate taxa, resulting in pathogen spillover.

In Homa Bay County, migratory birds and monitor lizards thrive on popular fishing activities in homesteads along Rusinga Island and may be involved in TBP transmission to humans and livestock. Though information on TBP's in the region is limited, an over two-decade-old study found Rhipicephalus appendiculatus transmitting T. parva to be highly prevalent among Zebu cattle grazing along the shores of Lake Victoria in Rusinga Island (25). In neighboring Siaya County, levels of recent human exposure to Rickettsia felis infection were found to be exceptionally high (>57\%) based on immunoglobulin G (IgG) seropositivity among febrile patients visiting a local health clinic, and Rickettsia africae has been isolated in Amblyomma variegatum $(26,27)$. A longitudinal study of cohorts of calves in Busia County found a high prevalence of tick-borne hemoparasites, mainly Theileria mutans (71.6\%), Theileria velifera (62.8\%), Anaplasma spp. Omatjenne (42.7\%), Anaplasma bovis (39.9\%), Theileria sp. (sable) (32.7\%), T. parva (12.9\%), and Theileria taurotragi (8.5\%) determined by reverse line blot hybridization assay (28). In Uganda, severe anaplasmosis, ECF, and babesiosis were reported a decade ago as causes of livestock morbidity and mortality around the Lake Victoria basin (29). More recently, high prevalences of diverse Theileria, Anaplasma, and Ehrlichia species have been identified among wildlife hosts in Lake Mburo National Park, Uganda (30) and Laikipia County, Kenya (31), and among ticks sampled in the Shimba Hills National Reserve, Kenya (32).

Intensification of tick and TBP surveillance, disease detection, and control of ticks are critical in informing public health decisions on mitigation, control, and early warning and response strategies in cases of disease outbreaks (33). To gain better insight into the diversity of ticks and TBPs parasitizing livestock, and the potential involvement of cohabitating reptiles in their epidemiology, within the Lake Baringo and Lake Victoria region ecosystems of Kenya, we utilized contemporary molecular biology techniques (32) to screen field-collected ticks sampled along the shores and adjacent islands in these regions. We report the presence and possible circulation of putative tick vectors of TBPs that are etiological agents of ehrlichiosis, anaplasmosis, rickettsiosis, babesiosis, and theileriosis of importance to livestock health and zoonotic diseases in SSA $(34,35)$.

\section{MATERIALS AND METHODS}

\section{Study Locality}

A TBP survey was conducted in 2012-2013 along the shores and adjacent islands of Lake Baringo and Lake Victoria in Kenya. 
Baringo County is located in the Great Rift Valley, $250 \mathrm{~km}$ northwest of Nairobi and covers $\sim 8,655 \mathrm{~km}^{2}$ in area. It has an average rainfall of $700 \mathrm{~mm}$ and altitude of $700 \mathrm{~m}$ above the sea level with average temperatures of $28^{\circ} \mathrm{C}$ (36). Three indigenous agro-pastoralists communities (Pokot, Tugen, and Njemps) inhabit Baringo County. They rely on livestock (mostly goats as well as cattle, sheep, and donkey) and irrigated crop production along the Perkerra, Molo, and Kerio rivers.

Homa Bay County lies within the Kenyan part of the Lake Victoria basin and covers $\sim 3,155 \mathrm{~km}^{2}$ in area. It has an a bimodal rainfall ranging between 250 and 1,650 mm per annum and an altitude of $970 \mathrm{~m}$ above the sea level with a mean average temperature range of 17.1 to $34.8^{\circ} \mathrm{C}(37)$. The "long rains" peak in April and the "short rains" in October. Most inhabitants belong to the Luo and Suba ethnic groups whose main socioeconomic activities are fishing and small-scale mixed farming, which includes keeping of livestock (cattle, sheep, and goats).

\section{Sampling}

Ethical clearance for the study was obtained from the Kenya Medical Research Institute ethics review committee (Approval Ref: non-SSC Protocol \#310) and sampling from wildlife was approved by the Kenya Wildlife Service Biodiversity Research and Monitoring committee (Permit Ref: KWS/BRM/5001). Informed oral consent was obtained from village elders on the study activities and from household heads before inclusion of their livestock in the study. Written consent could not be used due to low literacy levels and language barriers that required translation into local languages (Luo and Suba) among most of the community elders and livestock owners, hence oral consent was adopted to all for standardization. The Kenya Wildlife Services, Kenya's Directorate of Veterinary Services and Ministry of Health, were consulted before the study was initiated, and supervised oral consent and sampling. The oral consent was not documented since tick collection presented minimal risk to the livestock and involved no protocol for which written documentation is normally required. Before sampling, all animals were restrained manually in order to allow for tick collection. To minimize risks to livestock, animal sampling was carried out in a manner that addressed all pertinent animal welfare issues.

Homesteads were surveyed for livestock and reptiles parasitized with ticks in 2012 and 2013 during the wet months of April-May and October-December in both study areas close to human habitation. Those that had recently treated their animals with acaricides were excluded. Ticks were opportunistically sampled and pooled from goats (117), cattle (76), sheep (54), poultry houses (17), and dogs (15) found in selected homesteads, as well as tortoises (Stigmochelys pardalis) (18) and monitor lizards (Varanus niloticus) (4) that were common to homesteads in Baringo and Homa Bay counties, respectively. Up to 30 ticks were sampled per animal within each homestead and fully engorged ticks were not collected to minimize contamination from vertebrate host nucleic acids during extraction. Animals were manually restrained before plucking of live ticks from their bodies. Crocodiles were excluded because of high risk they pose.

Sampling was conducted in Baringo and Homa Bay counties alongside previously described mosquito sampling efforts (38-40) (Figure 1). In Baringo, we sampled in and adjacent to (i) Ruko Wildlife Conservancy, where livestock and humans live in close proximity to wildlife, (ii) Logumgum, a transmission hot spot for the 2006/2007 RVF outbreak (41), (iii) Kokwa, and (iv) Kampi ya Samaki, where locals keep relatively large numbers of livestock ( $\geq 20$ ). In Homa Bay County, Rusinga and Mbita areas were chosen due to previous studies in the area that detected the presence of T. parva antigens (42) and their association with reduced productivity of Zebu cattle under traditional management (43). Mfangano Island and Ngodhe offer sanctuary to a wide

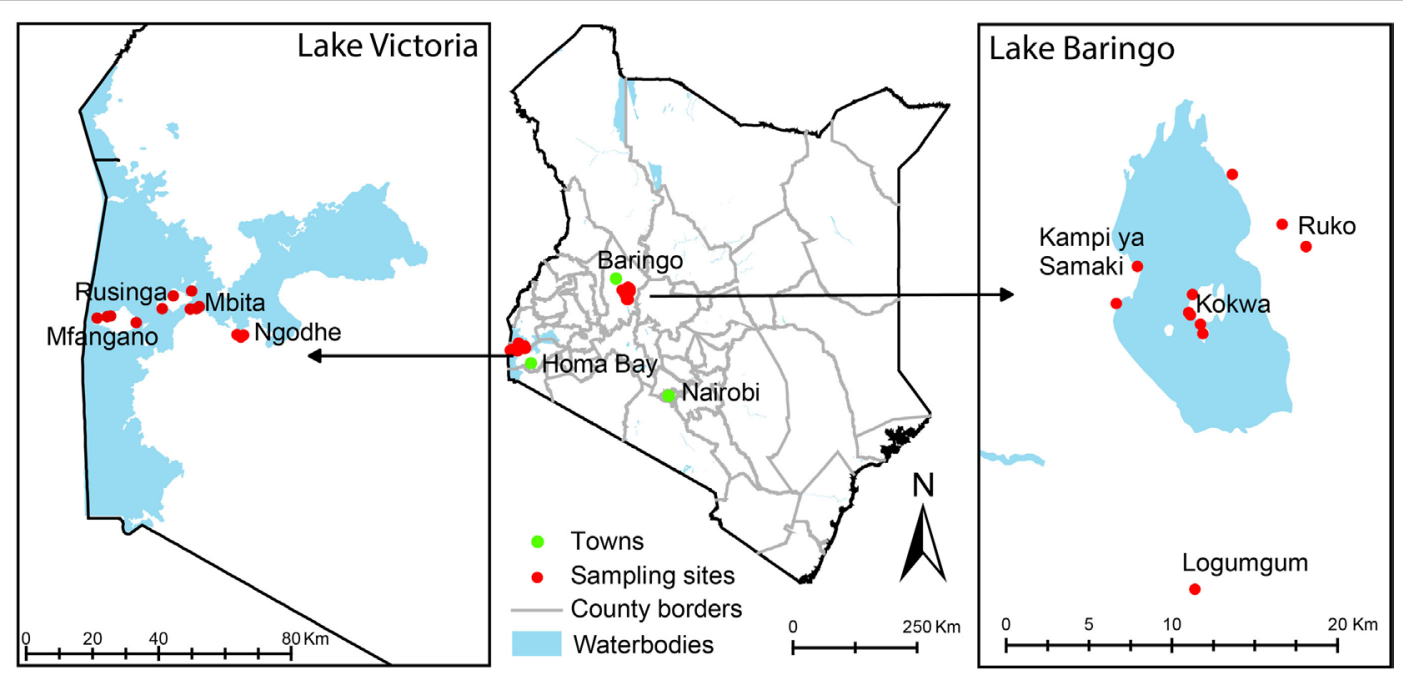

FIGURE 1 | Map of Kenya showing tick sampling areas in Kenya. Most of the sampling points were in rural villages where mosquito sampling has previously been described. 
diversity of wild birds that could be important in introductions of ticks to these areas.

After collection, ticks were frozen in liquid nitrogen and transported to the Martin Lüscher Emerging Infectious Diseases Laboratory at the International Centre of Insect Physiology and Ecology in Nairobi, where they were identified morphologically and sorted on a chilled surface (paper towels over $-80^{\circ} \mathrm{C}$ icepacks) under a dissecting microscope (Leica Microsystems, Buffalo Groove, Illinois). Ticks were identified morphologically to species level based on taxonomic keys of the genera Rhipicephalus (44), Amblyomma (45), Hyalomma, and Argas (46), developed by Walker and colleagues (47). Tick sorting entailed removal of highly engorged samples to reduce vertebrate host nucleic acids during extraction and pooling into groups of one to eight individuals by species, host, sampling site, and collection date.

\section{Molecular Identification of TBPs}

Tick pools were homogenized for $25 \mathrm{~s}$ in $1.5 \mathrm{ml}$ screw-cap tubes filled with zirconia/yttria-stabilized zirconium oxide beads (750 $\mathrm{mg}$ of $2.0 \mathrm{~mm}$ diameter and $150 \mathrm{mg}$ of $0.1 \mathrm{~mm}$ diameter) (Glen Mills, Clifton, NJ, USA) (48), $650 \mu$ l of phosphate-buffered saline using a Mini-Beadbeater-16 (BioSpec, Bartlesville, OK, USA). Total nucleic acids were extracted from these homogenates in an automated MagNa Pure 96 extraction system (Roche Diagnostics, Risch-Rotkreuz, Switzerland) using the small volume DNA/viral RNA kits (Roche Diagnostics). TBPs were detected and characterized by PCR followed by high-resolution melting (HRM) analyses. All gene fragments were amplified in an HRM capable RotorGene Q thermo cycler (QIAGEN, Hannover, Germany) to a final volume of $10 \mu$ l using HOT FIREPol EvaGreen HRM Mix (Solis BioDyne, Tartu, Estonia) and primers listed in Table 1. For identification of Ehrlichia and Anaplasma, we utilized previously described primers (49) of a short 16S rRNA amplicon $(<200 \mathrm{bp})$ and unresolved samples were subsequently analyzed by amplification and sequencing of longer (> 300bp) 16S rRNA fragments using the newly designed primer pairs, "Anaplasma long 16S rRNA Fwd/Rev" and "Ehrlichia long 16S rRNA Fwd/ Rev" (Table 1). For Rickettsia, we utilized the $r p m \mathrm{E}^{-\mathrm{tRNA}}{ }^{\mathrm{fMet}}$ intergenic spacer typing (50). The apicomplexan hemoparasites,
Theileria, Babesia and Hepatozoon, were amplified and resolved using previously described primers specific to the $18 \mathrm{~S}$ ribosomal gene (Table 1) (51).

The thermal cycling conditions used for amplification were as follows: initial denaturation at $95^{\circ} \mathrm{C}$ for $15 \mathrm{~min}$, followed by 10 cycles of $94^{\circ} \mathrm{C}$ for $20 \mathrm{~s}$, step-down annealing from $63.5^{\circ} \mathrm{C}$ decreasing by $1^{\circ} \mathrm{C}$ per cycle for $25 \mathrm{~s}$, and primer extension at $72^{\circ} \mathrm{C}$ for $30 \mathrm{~s}$; then 25 cycles of denaturation at $94^{\circ} \mathrm{C}$ for $25 \mathrm{~s}$, annealing at 50.5 for $20 \mathrm{~s}$, and extension at $72^{\circ} \mathrm{C}$ for $30 \mathrm{~s}$, followed by a final extension at $72^{\circ} \mathrm{C}$ for $7 \mathrm{~min}$. Following PCR, HRM profiles of amplicons were obtained through gradual increase in temperature from 75 to $90^{\circ} \mathrm{C}$ at $0.1^{\circ} \mathrm{C} / 2 \mathrm{~s}$ increments. Changes in fluorescence with time $(\mathrm{dF} / \mathrm{dT})$ were plotted against changes in temperature $\left({ }^{\circ} \mathrm{C}\right)$. Positive samples/amplicons were detected by observation of HRM curves and peaks. To identify the specific pathogen sequences associated with each unique HRM profiles, representative samples with single peaks for each of the profiles were purified with ExoSAP-IT PCR Product Cleanup kit (Affymetrix, Santa Clara, CA, USA) to remove unincorporated dNTPs and PCR primers before sequencing, which was outsourced from Macrogen (South Korea).

\section{Phylogenetic Analysis}

The returned sequences were edited and aligned, using the MAFFT (52) plugin in Geneious software version 8.1.4 (created by Biomatters) (53), with closely related sequences revealed by querying the GenBank nr database using the Basic Local Alignment Search Tool (54). Study sequences that were $>200$ bp were submitted to GenBank. From multiple alignments, some pathogens could not be fully characterized to species and were classified based on their genus. After visualizing neighborjoining phylogenetic trees (55) of the alignments, constructed within Geneious software, we constructed comparable maximum likelihood phylogenetic trees of the alignments, using PHYML v. 3.0 (56). The phylogenies employed the Akaike information criterion for automatic model selection and tree topologies were estimated using nearest neighbor interchange improvements over 1,000 bootstrap replicates. Phylogenetic trees were depicted using FIGTREE (57). Bacillus subtilis rpmE/tRNA ${ }^{\mathrm{fMet}}$

TABLE 1 | Primers used for detection of TBPs.

\begin{tabular}{|c|c|c|c|c|c|}
\hline Pathogen/gene target & Primer pair & $\begin{array}{l}\text { Amplicons size } \\
\text { (bp) }\end{array}$ & $\begin{array}{l}\text { Reference } \\
\text { sequence }\end{array}$ & Primer coordinates & Citation \\
\hline \multirow[t]{2}{*}{ Anaplasma short $16 \mathrm{~S}$ rRNA } & Fwd: GGGCATGTAGGCGGTTCGGT & $\sim 200$ & KJ410254 & $491-510$ & Tokarz et al. (49) \\
\hline & Rev: TCAGCGTCAGTACCGGACCA & & & $675-656$ & \\
\hline \multirow[t]{2}{*}{ Anaplasma long $16 \mathrm{~S}$ rRNA } & Fwd: CGGTGGAGCATGTGGTITAATTC & $\sim 330$ & & $853-875$ & Mwamuye et al. (32) \\
\hline & Rev: CGRCGTTGCAACCTATTGTAGTC & & & $1,183-1,161$ & \\
\hline \multirow[t]{2}{*}{ Ehrlichia short 16S rRNA } & Fwd: CGTAAAGGGCACGTAGGTGGACTA & $\sim 200$ & NR_074155 & $507-530$ & Tokarz et al. (49) \\
\hline & Rev: CACCTCAGTGTCAGTATCGAACCA & & & $701-678$ & \\
\hline \multirow[t]{2}{*}{ Ehrlichia long $16 \mathrm{~S}$ rRNA } & Fwd: GCAACCCTCATCCTTAGTTACCA & $\sim 400$ & & $1,045-1,067$ & Mwamuye et al. (32) \\
\hline & Rev: TGTTACGACTTCACCCTAGTCAC & & & $1,439-1,417$ & \\
\hline \multirow[t]{2}{*}{ Rickettsia/ompB } & For: GTAAAATTACCGGTAAGGGTTATAGC & $\sim 200$ & CP001612 & $1,020,788-1,020,813$ & Tokarz et al. (49) \\
\hline & Rev: ATACAAAGTGCTAATGCAACTGGG & & & $1,020,984-1,020,961$ & \\
\hline \multirow[t]{2}{*}{ Rickettsia rpmE/tRNA ${ }^{\mathrm{fmet}}$} & For: TCAGGTTATGAGCCTGACGA & $175-402$ & & $130,181-130,205$ & Zhu et al. (50) \\
\hline & Rev: TTCCGGAAATGTAGTAAATCAATC & & & $130,522-130,503$ & \\
\hline \multirow[t]{2}{*}{ Theileria/Babesia 18S rRNA } & Fwd: GAGGTAGTGACAAGAAATAACAATA & $\sim 500$ & HQ684067 & $330-354$ & Gubbels et al. (51) \\
\hline & Rev: TCTTCGATCCCCTAACTITC & & & $832-813$ & \\
\hline
\end{tabular}


(GenBank accession CP010434) and Hemolivia stellata 18S rRNA (GenBank accession KP881349) sequences were used as outgroups for the Rickettsia and apicomplexan hemoparasite phylogenies, respectively. The Ehrlichia/Anaplasma 16S rRNA phylogeny was midpoint rooted with the Paracoccus 16 S rRNA sequences as no suitable outgroup sequences were available in public databases. Midpoint rooting is appropriate for this phylogeny since the Paracoccus clade is distant to the Ehrlichial Anaplasma clade, ensuring consistency with outgroup rooting procedures (58).

\section{RESULTS}

\section{Tick Species Sampled}

A total of 585 tick pools comprised of 4,126 ticks of 14 species were collected and analyzed from both study areas. We sampled more ticks (80.47\%) in Baringo County (3,320 ticks in 456 tick pools) (Table 2), which had higher numbers of livestock per household $(>20)$, than in Homa Bay County (806 ticks in 129 tick pools) (Table 3 ), which had fewer $(<5)$ livestock per household, from diverse vertebrate hosts. In both study areas, most ticks were from goats, which were more heavily parasitized than the other animals sampled (Tables 2 and 3 ).

In Baringo County, 12 species were sampled from goats, sheep, cattle, poultry houses, dogs, and free ranging tortoises (Table 2), 11 of which were hard ticks (Family; Ixodidae) and one of which was a soft tick species (Argas persicus, 7.04\%) sampled from poultry houses in Kampi ya Samaki. Rhipicephalus pravus (44.88\%) sampled from domestic ruminants and dogs was the most frequent tick species identified, while Amblyomma falsomarmoreum (1.23\%) and Amblyomma nuttalli (0.66\%) sampled from free ranging tortoises at Kampi ya Samaki were the least frequent tick species identified.

In Homa Bay County, eight hard tick species were sampled from goats, sheep, cattle, dogs, and monitor lizards (Table 3). Rhipicephalus pravus (34.49\%), sampled from livestock and dogs, was the most frequent species identified, while Hyalomma truncatum $(2.23 \%)$ sampled from livestock was least frequent. Rhipicephalus appendiculatus (3.59\%), a known vector for ECF, and Amblyomma (Aponomma) latum (3.34\%), sampled from monitor lizards, were common only on Rusinga Island.

\section{TBPs Identified}

Tick-borne pathogen gene fragments with distinct HRM profiles (Figure 2) and representative sequences sharing $\geq 96 \%$ identity with a recognized TBP species, or $\geq 90 \%$ with a TBP genus on GenBank (Table 4), were detected in tick pools sampled in Baringo and Homa Bay counties (Table 5) from diverse individual vertebrate hosts (Table 6). In Baringo County, we detected sequences of agents of livestock or canine ehrlichiosis (E. ruminantium, Ehrlichia canis, and Ehrlichia sp.) (Figure 2A), anaplasmosis (Anaplasma ovis, Anaplasma platys, and A. bovis) (Figure 2B), rickettsiosis (Rickettsia aeschlimannii, Rickettsia

TABLE 2 | Ticks sampled from different host species in four study areas of Baringo County.

\begin{tabular}{|c|c|c|c|c|c|c|c|c|c|}
\hline Study area & Tick species & Pools & N (\%) & Cattle (\%) & Goats (\%) & Sheep (\%) & Dogs (\%) & Tortoises (\%) & Poultry (\%) \\
\hline Kampi Ya & Rh. pravus & 51 & 401 (12.07) & $82(2.47)$ & $186(5.6)$ & $95(2.86)$ & $38(1.14)$ & & \\
\hline \multirow[t]{7}{*}{ Samaki } & Ar. persicus & 34 & $234(7.04)$ & & & & & & $234(7.04)$ \\
\hline & Rh. pulchellus & 31 & $204(6.14)$ & $46(1.38)$ & $98(2.95)$ & $58(1.74)$ & $2(0.06)$ & & \\
\hline & Rh. evertsi evertsi & 17 & $133(4)$ & $24(0.72)$ & $66(1.98)$ & $38(1.14)$ & $5(0.15)$ & & \\
\hline & Am. variegatum & 5 & $33(0.99)$ & $19(0.57)$ & $5(0.15)$ & $9(0.27)$ & & & \\
\hline & Am. gemma & 4 & $17(0.51)$ & $8(0.24)$ & $6(0.18)$ & $3(0.09)$ & & & \\
\hline & Am. sparsum & 2 & $13(0.39)$ & $13(0.39)$ & & & & & \\
\hline & Am. nuttalli & 1 & $6(0.18)$ & & & & & $6(0.18)$ & \\
\hline Ruko & Rh. pravus & 31 & $235(7.07)$ & $52(1.56)$ & $128(2.85)$ & 55 (1.65) & & & \\
\hline \multirow[t]{9}{*}{ Conservancy } & Rh. evertsi evertsi & 28 & $219(6.59)$ & $59(1.77)$ & $46(1.38)$ & $68(2.04)$ & $46(1.38)$ & & \\
\hline & Rh. pulchellus & 27 & 209 (6.29) & $114(3.43)$ & 46 (1.38) & $30(0.9)$ & $19(0.57)$ & & \\
\hline & Hy. truncatum & 13 & 101 (3.04) & $52(1.56)$ & $14(0.42)$ & 35 (1.05) & & & \\
\hline & Am. variegatum & 8 & $52(1.56)$ & $41(1.23)$ & $6(0.18)$ & $5(0.15)$ & & & \\
\hline & Hy. rufipes & 7 & $47(1.41)$ & $28(0.84)$ & $7(0.21)$ & $6(0.18)$ & & & $6(0.18)$ \\
\hline & Am. gemma & 5 & $36(1.08)$ & $18(0.54)$ & $11(0.33)$ & $7(0.21)$ & & & \\
\hline & Am. sparsum & 4 & $32(0.96)$ & & & & & $32(0.96)$ & \\
\hline & Am. nuttalli & 2 & $16(0.48)$ & & & & & $16(0.48)$ & \\
\hline & Rh. praetextatus & 8 & $15(0.45)$ & $10(0.3)$ & $5(0.15)$ & & & & \\
\hline \multirow[t]{6}{*}{ Logumgum } & Rh. pravus & 97 & $731(22.01)$ & $161(4.85)$ & $304(9.15)$ & $202(6.08)$ & $64(1.92)$ & & \\
\hline & Am. variegatum & 26 & $187(5.83)$ & $121(3.64)$ & $23(0.69)$ & $43(1.29)$ & & & \\
\hline & Rh. evertsi evertsi & 14 & $110(3.31)$ & $25(0.75)$ & $24(0.72)$ & $15(0.45)$ & $46(1.38)$ & & \\
\hline & Am. gemma & 6 & $47(1.41)$ & $19(0.57)$ & $5(0.15)$ & $23(0.69)$ & & $41(1.23)$ & \\
\hline & Am. falsomarmoreum & 6 & $41(1.23)$ & & & & & $30(0.9)$ & \\
\hline & Am. sparsum & 5 & $30(0.9)$ & & & & & & \\
\hline \multirow[t]{4}{*}{ Kokwa } & Rh. pravus & 16 & $123(3.7)$ & $1(0.03)$ & $84(2.53)$ & $38(1.14)$ & & & \\
\hline & Rh. evertsi evertsi & 4 & $26(0.78)$ & $4(0.12)$ & $8(0.24)$ & $9(0.27)$ & $5(0.15)$ & & \\
\hline & Am. gemma & 4 & $22(0.66)$ & $2(0.06)$ & $12(0.36)$ & $8(0.24)$ & & & \\
\hline & Total & 456 & 3,320 & 899 (27.07) & $1,084(32.56)$ & 747 (22.5) & $225(6.77)$ & $125(3.76)$ & $240(7.22)$ \\
\hline
\end{tabular}

Percentages are out of the total number of ticks sampled. N, number of ticks sampled. 
TABLE 3 | Ticks sampled from different host species in four study areas of Homa Bay County.

\begin{tabular}{|c|c|c|c|c|c|c|c|c|}
\hline Study Area & Tick species & Pools & N (\%) & Cattle (\%) & Goats (\%) & Sheep (\%) & Dogs (\%) & Monitor lizards (\%) \\
\hline \multirow[t]{4}{*}{ Ngothe } & Rh. pulchellus & 9 & $61(7.56)$ & $24(2.97)$ & $23(2.85)$ & $14(1.73)$ & & \\
\hline & Rh. evertsi evertsi & 8 & $45(5.58)$ & $3(0.37)$ & $30(2.72)$ & $4(0.49)$ & 8 (2.23) & \\
\hline & Am. variegatum & 4 & $18(2.23)$ & $9(1.11)$ & $4(0.49)$ & $5(0.62)$ & & \\
\hline & Hy. truncatum & 3 & $12(1.48)$ & $9(1.11)$ & & $3(0.37)$ & & \\
\hline \multirow[t]{5}{*}{ Mbita } & Rh. pravus & 13 & 83 (10.29) & $13(1.61)$ & $38(4.71)$ & $28(2.47)$ & $4(0.49)$ & \\
\hline & Am. gemma & 9 & $67(8.31)$ & $51(6.32)$ & $12(1.48)$ & $4(0.49)$ & & \\
\hline & Rh. evertsi evertsi & 7 & $40(4.96)$ & $11(1.36)$ & $18(2.23)$ & $6(0.74)$ & $5(0.62)$ & \\
\hline & Am. Variegatum & 5 & 32 (3.97) & $16(1.98)$ & $6(0.74)$ & $10(1.24)$ & & \\
\hline & Rh. pulchellus & 1 & $6(0.74)$ & $6(0.74)$ & & & & \\
\hline \multirow[t]{4}{*}{ Mfangano } & Rh. pravus & 14 & $94(11.66)$ & $18(2.23)$ & 37 (4.59) & 27 (2.35) & $12(1.48)$ & \\
\hline & Am. variegatum & 6 & $45(5.58)$ & $25(3.1)$ & 14(1.73) & $6(0.74)$ & & \\
\hline & Rh. pulchellus & 8 & $41(5.08)$ & $9(1.11)$ & $19(2.35)$ & $13(1.61)$ & & \\
\hline & Rh. evertsi evertsi & 5 & 26 (3.22) & $5(0.62)$ & $14(1.73)$ & $4(0.49)$ & $3(0.37)$ & \\
\hline \multirow[t]{7}{*}{ Rusinga } & Rh. pravus & 14 & $101(12.53)$ & $15(1.86)$ & $57(7.07)$ & $15(1.86)$ & $14(1.73)$ & \\
\hline & Rh. pulchellus & 7 & $50(6.2)$ & $12(1.48)$ & $26(3.22)$ & $11(1.36)$ & $1(0.12)$ & \\
\hline & Rh. appendiculatus & 5 & 29 (3.59) & $25(3.1)$ & $4(0.49)$ & & & \\
\hline & Am. latum & 4 & 27 (3.34) & & & & & 27 (3.34) \\
\hline & Rh. evertsi evertsi & 6 & $23(2.85)$ & $4(0.49)$ & $11(1.36)$ & $2(0.24)$ & $6(0.74)$ & \\
\hline & Hy. truncatum & 1 & $6(0.74)$ & $6(0.74)$ & & & & \\
\hline & Total & 129 & 806 & 261 (32.38) & 307 (38.08) & 152(18.85) & $53(6.57)$ & $27(3.34)$ \\
\hline
\end{tabular}

Percentages are out of the total number of ticks sampled. N, Number of ticks sampled.
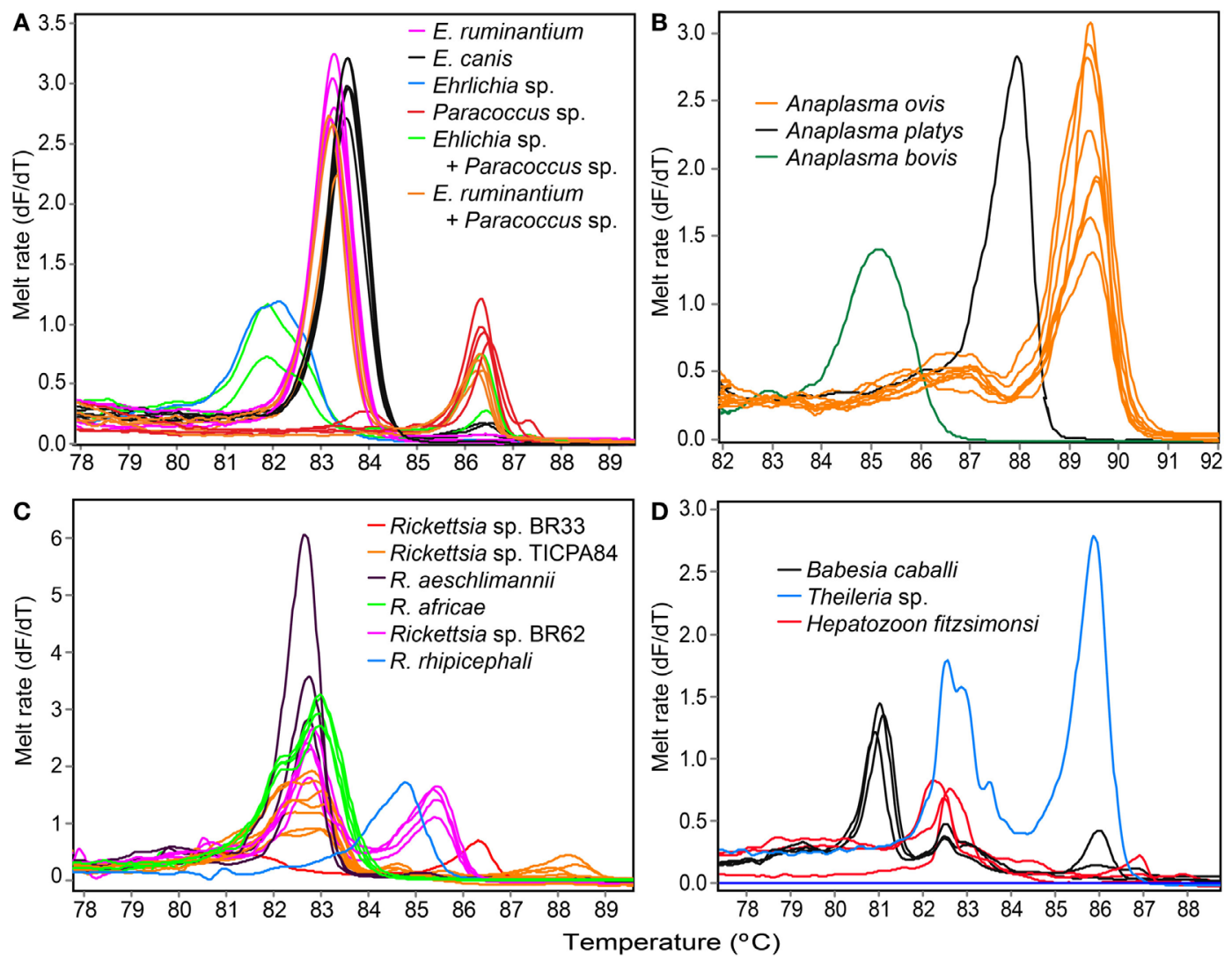

FIGURE 2 | Melting rate profiles of tick-borne pathogens in field collected tick samples. PCR amplicon melt rates are represented as change in fluorescence with increasing temperature (dF/dT) of (A) Ehrlichia spp. and Paracoccus sp. 16S rRNA, (B) Anaplasma 16S rRNA, (C) Rickettsia rpmE/tRNA ${ }^{\mathrm{fMet}}$, and (D) apicomplexan hemoparasite 18S rRNA gene segments. 
TABLE 4 | Nucleic acid sequence identities of tick-borne pathogens detected in Baringo and Homa Bay counties.

\begin{tabular}{|c|c|c|c|c|c|c|}
\hline $\begin{array}{l}\text { Pathogen } \\
\text { detected }\end{array}$ & Tick species & Study areas & $\begin{array}{l}\text { Reference } \\
\text { GenBank } \\
\text { Accessions }\end{array}$ & $\begin{array}{l}\text { Percentage } \\
\text { identity (17 } \\
\text { November } 2016 \\
\text { e-value) }\end{array}$ & Locus & $\begin{array}{l}\text { Sequence } \\
\text { length, bp } \\
\text { (GenBank } \\
\text { Accession) }\end{array}$ \\
\hline \multirow[t]{2}{*}{ Anaplasma bovis } & $\begin{array}{l}\text { Am. gemma, Am. variegatum, Rh. evertsi evertsi, and } \\
\text { Hy. truncatum }\end{array}$ & Logumgum and Ruko & U03775 & $99(1 e-135)$ & $\begin{array}{c}\text { Short } \\
16 S\end{array}$ & 185 \\
\hline & Hy. rufipes, Rh. praetextatus & & & $99(1 e-163)$ & $\begin{array}{l}\text { Long } \\
16 S\end{array}$ & 320 (KT266580) \\
\hline \multirow[t]{2}{*}{ A. ovis } & $\begin{array}{l}\text { Am. variegatum, Rh. pulchellus, Am. gemma, and } \\
\text { Rh. appendiculatus }\end{array}$ & $\begin{array}{l}\text { Logumgum, Kampi Ya } \\
\text { Samaki, and Rusinga }\end{array}$ & KJ410245 & $99(1 e-135)$ & $\begin{array}{c}\text { Short } \\
16 S\end{array}$ & 185 \\
\hline & & & & $100(8 e-170)$ & $\begin{array}{l}\text { Long } \\
16 S\end{array}$ & 328 (KT266581) \\
\hline A. platys & Rh. evertsi, Rh. pulchellus, and Rh. pravus & $\begin{array}{l}\text { Ngothe, Mfangano, } \\
\text { Kampi ya Samaki, and } \\
\text { Kokwa }\end{array}$ & LC018183 & $100(5 e-162)$ & $\begin{array}{l}\text { Short } \\
16 S\end{array}$ & 185 \\
\hline $\begin{array}{l}\text { Ehrlichia } \\
\text { ruminantium }\end{array}$ & $\begin{array}{l}\text { Am. gemma, Am. variegatum, Am. sparsum, and } \\
\text { Am. evertsi evertsi }\end{array}$ & Ruko and Logumgum & NR_074155 & $99(1 e-94)$ & $\begin{array}{c}\text { Short } \\
16 S\end{array}$ & 194 \\
\hline $\begin{array}{l}\text { E. (Cowdria) } \\
\text { ruminantium }\end{array}$ & Am. gemma, Am. variegatum, and $R h$. evertsi evertsi & $\begin{array}{l}\text { Ngothe, Mfangano, and } \\
\text { Mbita }\end{array}$ & U03776 & $99(6 e-93)$ & $\begin{array}{l}\text { Short } \\
16 S\end{array}$ & 194 \\
\hline \multirow[t]{2}{*}{ E. canis } & $\begin{array}{l}\text { Rh. pravus, Rh. evertsi evertsi, Rh. pulchellus, and } \\
\text { Am. latum }\end{array}$ & $\begin{array}{l}\text { Logumgum, Rusinga, } \\
\text { and Mbita }\end{array}$ & CP000107 & $\begin{array}{l}100(3 e-96) 100 \\
(0.0)\end{array}$ & $\begin{array}{c}\text { Short } \\
16 S\end{array}$ & 194 \\
\hline & & & & & $\begin{array}{l}\text { Long } \\
16 S\end{array}$ & 555 (KT266591) \\
\hline \multirow[t]{2}{*}{$\begin{array}{l}\text { Ehrlichia sp. } \\
\text { (Tibet/Xinjiang) }\end{array}$} & Rh. pulchellus, Am. gemma, and Am. variegatum & Ruko & $\begin{array}{l}\text { AF414399, } \\
\text { JX402605 }\end{array}$ & $98(3 e-96)$ & $\begin{array}{l}\text { Short } \\
16 S\end{array}$ & 194 \\
\hline & Rh. evertsi evertsi, Rh. pulchellus, and Rh. praetextatus & Ruko and Kokwa Island & & $100(0.0)$ & $\begin{array}{l}\text { Long } \\
16 S\end{array}$ & 384 (KT266592) \\
\hline Paracoccus sp. & $\begin{array}{l}\text { Am. variegatum, Am. gemma, Am. sparsum, and } \\
\text { Am. falsomarmoreum }\end{array}$ & Logumgum & KP003988 & $97(6 e-88)$ & $\begin{array}{c}\text { Short } \\
16 S\end{array}$ & 151 \\
\hline Rickettsia africae & $\begin{array}{l}\text { Am. gemma, Am. variegatum, Am. sparsum, and } \\
\text { Rh. evertsi evertsi }\end{array}$ & $\begin{array}{l}\text { Kampi Ya Samaki, } \\
\text { Ruko, and Logumgum }\end{array}$ & CP001612 & $100(3 e-143)$ & tRNA & 280 (KT266590) \\
\hline R. rhipicephali & Rh. evertsi evertsi and Rh. pulchellus & Kampi ya Samaki, Ruko & CP013133 & $99(2 \mathrm{e}-176)$ & tRNA & 343 (KT266586) \\
\hline R. aeschlimannii & Hy. truncatum, Hy. rufipes, and Rh. pulchellus & $\begin{array}{l}\text { Kokwa, Logumgum, } \\
\text { and Kampi ya Samaki }\end{array}$ & HQ335165 & $100(1 e-178)$ & tRNA & 344 (KT266585) \\
\hline Rickettsia sp. BR62 & Rh. pulchellus and Rh. evertsi evertsi & Ruko & AP011532 & $94(7 e-115)$ & tRNA & 281 (KT266587) \\
\hline $\begin{array}{l}\text { Rickettsia sp. } \\
\text { TICPA84 }\end{array}$ & Am. gemma and Rh. evertsi evertsi & Logumgum and Kokwa & CP013133 & $99(2 \mathrm{e}-171)$ & tRNA & 343 (KT266588) \\
\hline Rickettsia sp. BR33 & Rh. pulchellus & Ruko & KR492955 & $97(1 e-52)$ & tRNA & 354 (KT266589) \\
\hline Babesia caballi & Rh. pulchellus & Kampi ya Samaki & EU642514 & $98(1 e-122)$ & $18 S$ & $\begin{array}{c}255 \mathrm{bp} \\
\text { (KT266583) }\end{array}$ \\
\hline Theileria sp. & Rh. evertsi evertsi & Logumgum & AF245279 & $90(3 e-140)$ & $18 S$ & 402 (KT266584) \\
\hline $\begin{array}{l}\text { Hepatozoon } \\
\text { fitzsimonsi }\end{array}$ & Am. falsomarmoreum and Am. sparsum & Logumgum and Ruko & KR069084 & $100(0.0)$ & $18 S$ & 438 (KT266582) \\
\hline
\end{tabular}

rhipicephali, Rickettsia africae, and Rickettsia spp.) (Figure 2C), as well as Babesia caballi, Hepatozoon fitzsimonsi, Theileria sp. (Figure 2D), and Paracoccus sp. (Figure 2A; Table 5). However, in Homa Bay County, we only detected E. ruminantium, E. canis, A. ovis, and A. platys (5). The maximum likelihood phylogenies of all sequences obtained among previously characterized, closely related TBPs are represented in Figures 3-5.

Among Ehrlichia sequences identified, E. ruminantium sequences from Baringo shared $99 \%$ nucleotide sequence identity with GenBank accession NR_074155 (59), whereas those from Homa Bay County were more similar (99\% identity) to an $E$. (Cowdria) ruminantium sequence (GenBank accession U03776) (60) (Table 4). In both sampling areas, these were detected in Am. variegatum, Amblyomma gemma, and Rhipicephalus evertsi evertsi tick pools (Table 5) from livestock and dogs (Tables 2, 3 and 6). Surprisingly, E. ruminantium was also detected in Am. falsomarmoreum (four pools) and Am. nuttalli (three pools) ticks (Table 5) sampled from tortoises (Tables 2 and 6) and, more importantly, Am. sparsum ticks (Table 5) sampled from both tortoises (five pools) and cattle (two pools) in Logumgum and Kampi ya Samaki, respectively, in Baringo County (Tables 2 and 6). Ehrlichia canis sequences (submitted GenBank accession KT266591) (Table 4) were detected in $R h$. pravus, $R h$. evertsi evertsi, and Rhipicephalus pulchellus tick pools (Table 5) sampled from livestock and domestic dogs of Logumgum in Baringo County (Tables 2 and 6), Rh. pulchellus ticks (Table 5) sampled from cattle in Mbita (Tables 3 and 6), and notably, Am. (aponomma) latum ticks (Table 5) parasitizing monitor lizards in Rusinga Island (Tables 3 and 6) of Homa Bay County. Ehrlichia sp. sequences (submitted GenBank accession KT266592) sharing 98-100\% identity with Ehrlichia spp. isolates from Tibet and Xinjiang, China (GenBank accessions AF414399, JX402605) (61) (Table 4) were detected in Am. gemma (7 pools), Am. variegatum (3 pools), Rh. evertsi evertsi (13 pools), Rh. pulchellus (9 pools), 


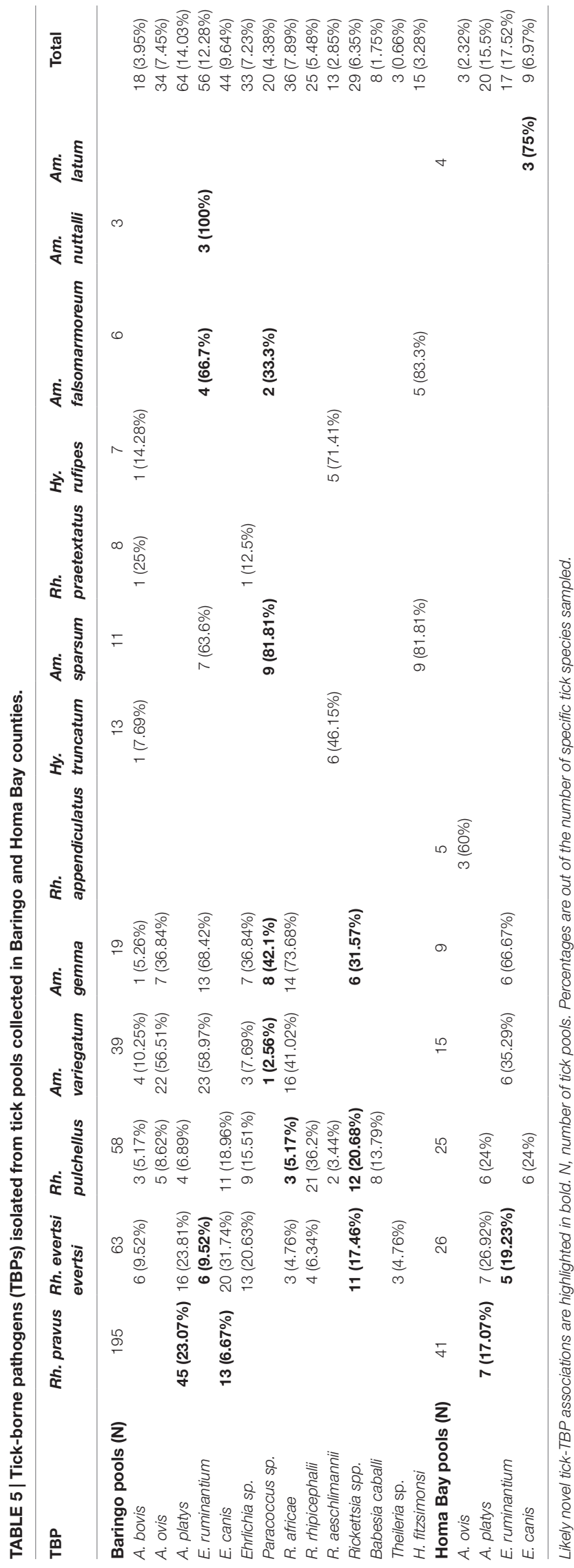

and $R h$. praetextatus (1 pool) ticks (Table 5) sampled from livestock in Ruko, Kokwa Island, and Logumgum areas of Baringo County (Tables 2 and 6).

We also amplified 195 bp Paracoccus sp. sequences using the "Ehrlichia short $16 \mathrm{~S}$ rRNA" primers (Table 4) in Am. variegatum (one pool), Am. sparsum (nine pools), Am. falsomarmoreum (two pools), and Am. gemma (eight pools) (Table 5) ticks sampled from livestock and tortoises (Tables 2 and 6). There were instances in which we detected both Paracoccus sp. and E. ruminantium within single tick pools as illustrated by double HRM peaks (Figure 2A). Six pools of Am. sparsum and four of Am. variegatum had both E. ruminantium and Paracoccus sp., while three pools of Am. variegatum had a mixed infection of Ehrlichia sp. and Paracoccus sp. (Figure 2A).

The $A$. ovis sequences (submitted GenBank accession KT266581) (Table 4) were detected in Am. variegatum (22 pools), Am. gemma (7 pools), and Rh. pulchellus (5 pools) ticks (Table 5) parasitizing livestock in Logumgum and Kampi ya Samaki of Baringo County (Tables 2 and 6) and in Rh. appendiculatus (3 pools) (Table 5) parasitizing goats and cattle in Rusinga Island of Homa Bay County (Tables 3 and 6). Anaplasma platys sequences (Table 4) were detected from Rhipicephalus tick pools, including $R h$. evertsi evertsi ( 15 pools), Rh. pravus (45 pools), and Rh. pulchellus (4 pools) (Tables 5 and 6) sampled from domesticated dogs in Ruko Conservancy, Kampi ya Samaki, Ngodhe, Mbita, Mfangano, and Rusinga Island study areas (Tables 2, 3 and 6). Anaplasma bovis sequences (submitted GenBank accession KT266580) (Table 4) were detected in 18 pools of Amblyomma ticks. Hyalomma and Rhipicephalus ticks (Table 5) sampled from livestock of Logumgum and Ruko study areas (Tables 2 and 6).

The $R$. aeschlimannii pathogen sequences (submitted GenBank accession KT266585) (Table 4) were detected in Hy. truncatum (6 pools), Hyalomma rufipes (5 pools), and Rh. pulchellus (2 pools) ticks (Table 5) sampled from livestock in Logumgum, Kampi ya Samaki, and Kokwa Island (Tables 2 and 6). Rickettsia rhipicephali sequences (submitted GenBank accession KT266586) (Table 4) were amplified in Rh. evertsi evertsi (4 pools) and Rh. pulchellus (21 pools) ticks (Table 5) sampled from goats, sheep, cattle, and dogs at Kampi ya Samaki, Logumgum, and Ruko Conservancy of Baringo County (Tables 2 and 6). Rickettsia africae sequences (submitted GenBank accession KT266590) (Table 4) were detected in Am. gemma (14 pools), Am. variegatum (16 pools), Am. sparsum (3 pools), and Rh. evertsi evertsi (3 pools) (Table 5) parasitizing livestock in Kampi ya Samaki, Logumgum, and Ruko Conservancy of Baringo County (Tables $\mathbf{2}$ and 6). We also obtained three novel Rickettsia spp. sequences from Baringo County tick pools. The Rickettsia sp. BR62 (submitted GenBank accession KT266587) and BR33 (submitted GenBank accession KT266589) sequences (Table 4) were detected in Rh. pulchellus (12 pools) and Rh. evertsi evertsi (9 pools) ticks (Table 5) sampled from goats in Ruko Conservancy (Tables 2 and 6). Rickettsia sp. TICPA84 sequences (submitted GenBank accession KT266588) were detected in Am. gemma (six pools) from Logumgum and Rh. evertsi evertsi (three pools) from livestock and dogs (Tables 5 and 6) in Kokwa Island (Table 2).

Among apicomplexan hemoparasites, we detected B. caballi sequences (submitted GenBank accession KT266583) (Table 4) 
TABLE 6 | Vertebrate hosts from which ticks with tick-borne pathogens (TBPs) were isolated in the two study regions.

\begin{tabular}{|c|c|c|c|c|c|c|}
\hline TBP & Cattle (\%) & Goat (\%) & Sheep (\%) & $\operatorname{Dog}(\%)$ & Tortoise & Monitor lizard (\%) \\
\hline \multicolumn{7}{|l|}{ Baringo County } \\
\hline $\mathrm{N}$ (vertebrate hosts) & 45 & 87 & 33 & 9 & 18 & 0 \\
\hline Anaplasma bovis & $8(17.8)$ & $6(6.8)$ & $3(9.1)$ & & & \\
\hline A. ovis & $7(15.6)$ & $5(5.7)$ & $10(30.3)$ & & & \\
\hline A. platys & & $17(19.5)$ & & $9(100)$ & & \\
\hline Ehrlichia ruminantium & $17(37.8)$ & $14(16.1)$ & $3(9.1)$ & & $12(66.7)$ & \\
\hline E. canis & & $15(17.2)$ & & $9(100)$ & & \\
\hline Ehrlichia sp. & $11(24.4)$ & $6(6.9)$ & $6(18.2)$ & $2(13.3)$ & & \\
\hline Paracoccus sp. & $5(11.1)$ & $8(9.2)$ & $3(9.1)$ & & $3(16.7)$ & \\
\hline Rickettsia africae & $17(37.8)$ & $6(6.8)$ & $11(33.3)$ & & & \\
\hline R. rhipicephali & $8(17.7)$ & $4(3.4)$ & $5(15.2)$ & $2(22.2)$ & & \\
\hline R. aeschlimannii & $5(11.1)$ & $2(2.3)$ & $6(18.2)$ & & & \\
\hline Rickettsia sp. BR62 & & $5(5.7)$ & & & & \\
\hline Rickettsia sp. TICPA84 & $3(6.7)$ & $2(2.3)$ & 5 (15.2) & $2(22.2)$ & & \\
\hline Rickettsia sp. BR33 & & $2(2.3)$ & & & & \\
\hline Babesia caballi & $8(17.7)$ & & & & & \\
\hline Theileria sp. & $3(6.7)$ & & & & & \\
\hline Hepatozoon fitzsimonsi & & & & & $14(77.8)$ & \\
\hline \multicolumn{7}{|l|}{ Homa Bay County } \\
\hline N (vertebrate hosts) & 31 & 30 & 21 & 7 & 0 & 4 \\
\hline A. ovis & $1(3.2)$ & 1 (3.3) & $1(4.8)$ & & & \\
\hline A. platys & $4(12.9)$ & $2(6.6)$ & $3(14.3)$ & $4(57.1)$ & & \\
\hline E. (Cowdria) ruminantium & $9(29.0)$ & $4(13.3)$ & & & & \\
\hline E. canis & $1(3.2)$ & & & $4(57.1)$ & & $4(100)$ \\
\hline
\end{tabular}

Likely novel vertebrate host-TBP associations are highlighted in bold. N, number of vertebrate hosts.

Percentages are out of the number of specific vertebrate host species sampled.

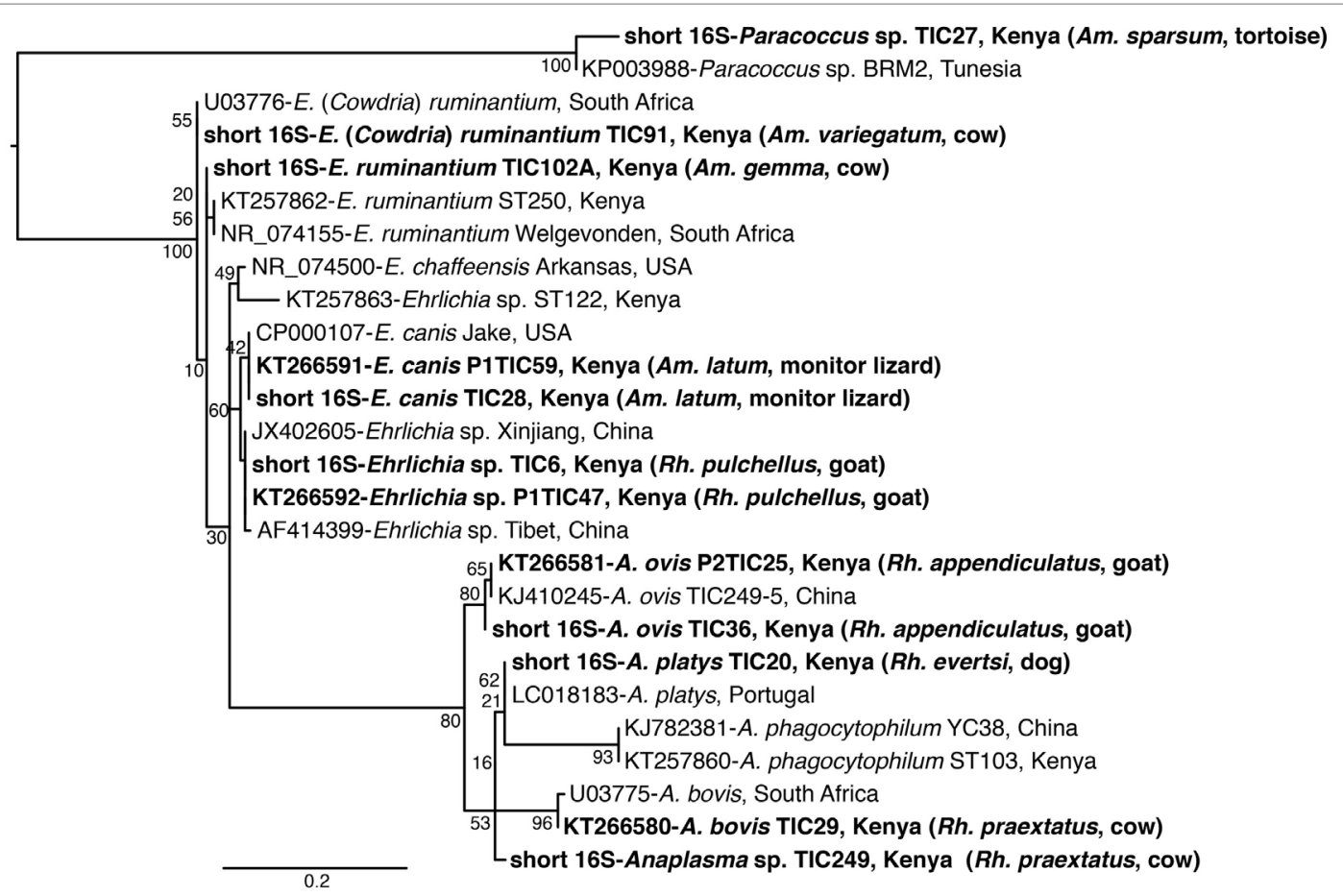

FIGURE 3 | Maximum likelihood phylogenetic analysis of 16S rRNA gene fragments of Anaplasma, Ehrlichia, and Paracoccus sequences identified with related sequences. GenBank accession numbers, species identifications, isolates, and country of origin are indicated for each $16 S$ rRNA gene sequence. Sequences from this study are in bold with tick and vertebrate host species associated with the study isolates indicated in brackets. Bootstrap values at the major nodes are of percentage agreement among 1,000 replicates. The branch length scale represents substitutions per site. 


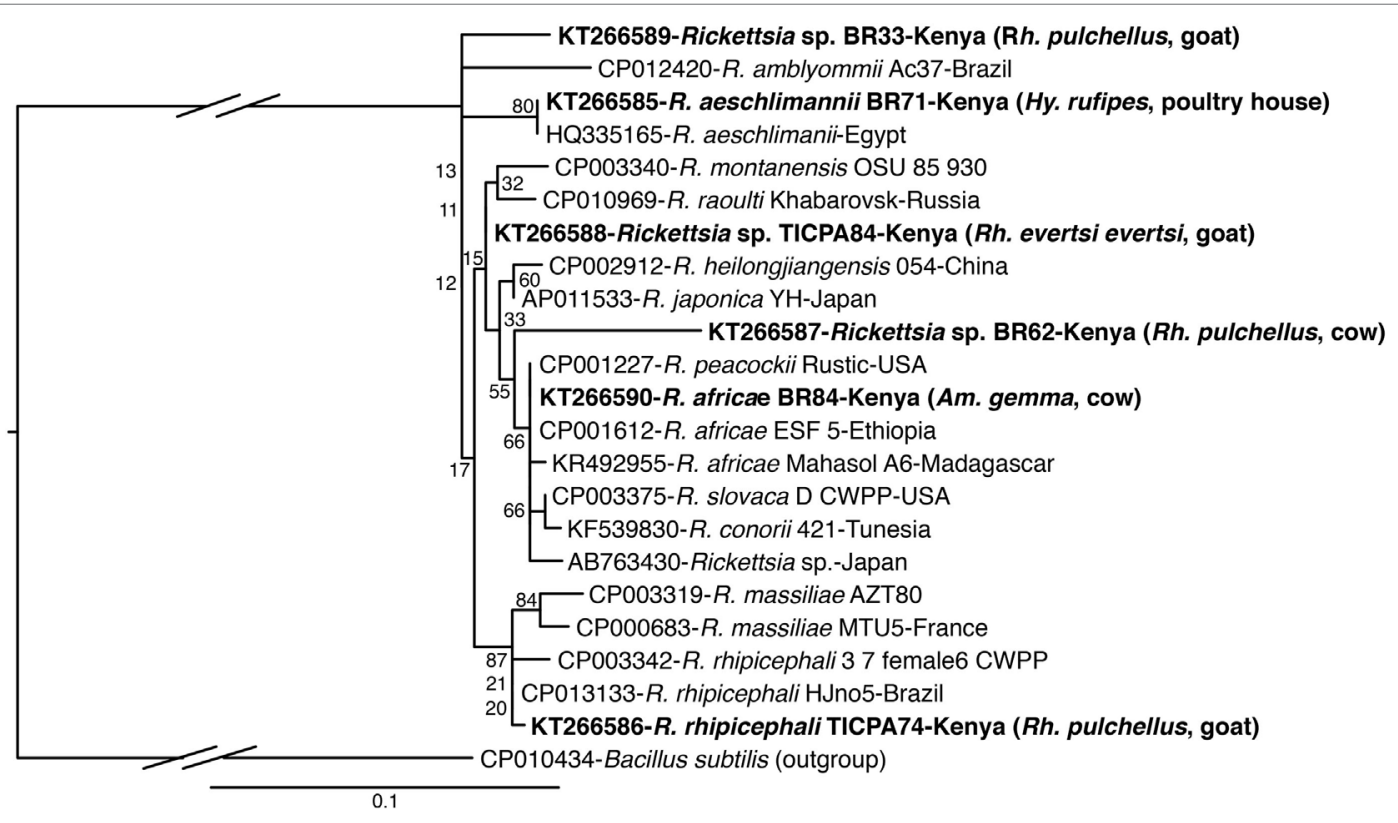

FIGURE 4 | Maximum likelihood phylogenetic analysis of rpmE/tRNA ${ }^{\text {fMet }}$ gene fragments of Rickettsia sequences identified with related sequences. GenBank accession numbers, species identifications, isolates, and country of origin are indicated for each 16S rRNA gene sequence. Sequences from this study are in bold with tick and vertebrate host species associated with the study isolates indicated in brackets. Bootstrap values at the major nodes are of percentage agreement among 1,000 replicates. The branch length scale represents substitutions per site. The gaps indicated in the branches to the Bacillus subtilis outgroup represent 1.2 substitutions per site.

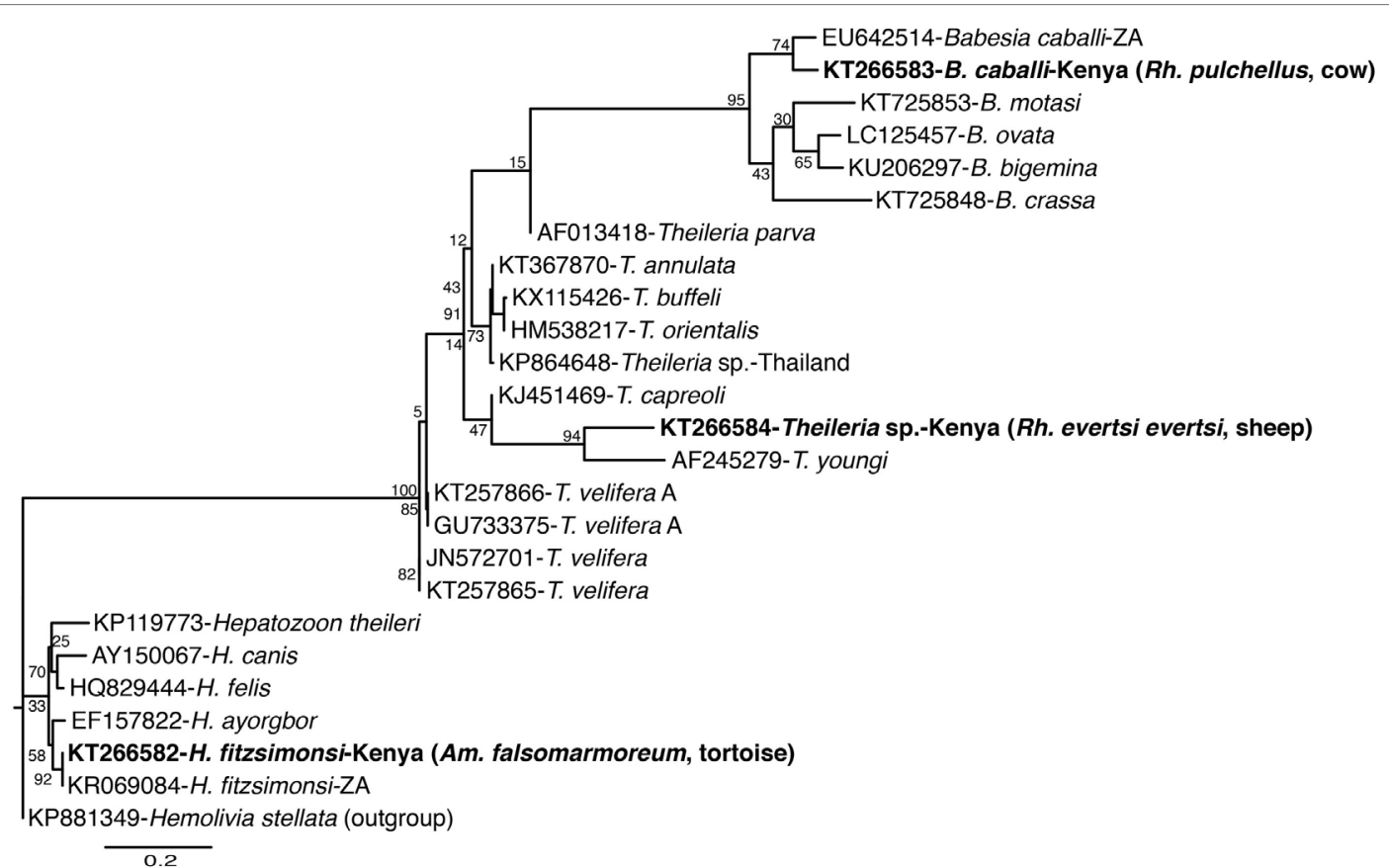

FIGURE 5 | Maximum likelihood phylogenetic analysis of 18S rRNA gene fragments of Apicomplexa (Hepatozoon, Babesia, and Theileria) sequences identified with related sequences. GenBank accession numbers, species identifications, isolates, and country of origin are indicated for each 16S rRNA gene sequence. Sequences from this study are in bold with tick and vertebrate host species associated with the study isolates indicated in brackets. Bootstrap values at the major nodes are of percentage agreement among 1,000 replicates. The branch length scale represents substitutions per site. 
in Rh. pulchellus (eight pools) ticks (Table 5) parasitizing cattle in Kampi ya Samaki, Baringo County (Tables 2 and 6). Hepatozoon fitzsimonsi sequences (submitted GenBank accession KT266582) (Table 4) were amplified in Am. sparsum (nine pools) and Am. falsomarmoreum (five pools) ticks (Table 5) sampled from free ranging tortoises in Baringo County (Tables 2 and 6). Theileria sp. sequences (submitted GenBank accession KT266584) (Table 4) were also amplified in Rh. evertsi evertsi (three pools) ticks (Table 5) parasitizing cattle at Logumgum in Baringo County (Tables 2 and 6).

\section{DISCUSSION}

We sampled 13 tick species that are potential vectors of ehrlichiosis, anaplasmosis, rickettsiosis, theileriosis, and babesiosis from livestock, poultry houses, and reptiles in domestic surroundings of Baringo and Homa Bay Counties. Most tick species were taken from multiple host taxa, except for Hy. truncatum that was restricted to cattle, Ar. persicus that was restricted to poultry, Am. latum that was restricted to monitor lizards, and Am. nuttalli and Am. falsomarmoreum that were both restricted to tortoises. Overall, we identified 14 TBPs, most from multiple tick species and predominantly from Baringo County samples. These included novel Ehrlichia sp., Rickettsia sp., Paracoccus sp., and Theileria sp. sequences that warrant further investigations into their potential pathogenicity. Most interestingly, Am. sparsum ticks infected with E. ruminantium, the causative agent of heartwater ehrlichiosis, were sampled from both tortoises and cattle. The complex pathogen-tick-host relationships presented here are important to public health in mitigating TBP transmission and possible associated disease outbreaks in these foci, as well as other areas of Kenya, with wider geographical implications.

Heartwater ehrlichiosis is an important rickettsial disease of wildlife and livestock ruminants in SSA, with considerable economic impact (62). Unlike past studies, which found E. ruminantium to be specific to Amblyomma tick species $(63,64)$, we also isolated the pathogen from $R h$. evertsi evertsi sampled from livestock in both study locations, though we cannot rule out that this may have come from livestock blood meals. Amblyomma variegatum Fabricius 1794 is the most common and widely distributed Amblyomma tick species of livestock in SSA and is a very important vector of the E. ruminantium in cattle (65). Amblyomma gemma Dönitz 1909 sampled from livestock in both study areas was once thought to be less important as a disease vector, but has since been linked with the transmission of a number of pathogens $(65,66)$. Laboratory studies have also shown that Am. gemma can transmit E. ruminantium from infected African buffalo to sheep (67). In this study, we detected E. ruminantium in 58.97 and $35.29 \%$ of Am. variegatum tick pools and in 68.42 and $66.67 \%$ of Am. gemma tick pools sampled in Baringo and Homa Bay Counties, respectively, suggesting very high infection rates. However, due to the pooled approach used to identify TBP's, these infection percentages do not provide a true estimate of infection rates.

Most importantly, E. ruminantium was also detected in Am. sparsum samples from both cattle and tortoises in Baringo as well as in Am. falsomarmoreum and Am. nuttalli found exclusively on tortoises. The occurrence of E. ruminantium has previously been reported among Am. sparsum that were sampled from tortoises imported into the United States from Zambia (14). Amblyomma nuttalli and Am. falsomarmoreum have both been associated with tortoises before $(68,69)$, but no report that we are aware of has implicated them with harboring or transmitting of agents of heartwater ehrlichiosis. Our findings suggest that $E$. ruminantium may be potentially transmitted between cattle and tortoises by Am. sparsum ticks and within tortoise populations by Am. nuttalli and Am. falsomarmoreum ticks. Similarly, we found E. canis, the causative agent of canine ehrlichiosis, in Am. latum ticks taken from monitor lizards in Homa Bay County. These pathogen-tick-host associations suggest complex transmission dynamics in the epidemiology of heartwater in Baringo County and canine ehrlichiosis in Homa Bay County that potentially involve reptilian reservoir hosts that are rarely considered in epidemiological studies of these pathogens.

From domestic ruminants and dogs, $R h$. pravus Dönitz 1910 was the most frequent tick parasite sampled in Baringo and Homa Bay counties, followed by Rh. evertsi evertsi and Rh. pulchellus. Each of these three tick species were found parasitizing dogs, with pools infected with canine anaplasmosis (A. platys) and ehrlichiosis (E. canis), the latter only in Baringo. Rhipicephalus pravus is considered the most common tick species among domestic and wildlife animals in Kenya (70-72), but has rarely been implicated in pathogen transmission. These findings potentially incriminate $R h$. pravus in the transmission of both A. platys and E. canis. Further studies to determine its vector competence will be critical to understanding its role in TBP transmission.

We detected $A$. bovis in multiple species of each of the tick genera (Rhipicephalus, Amblyomma, and Hyalomma) parasitizing livestock in Baringo County, but none in Homa Bay. The highest rates of $A$. bovis detection occurred in $R h$. praetextatus Gerstäcker 1873, a three-host tick species that was sampled from cattle and goats in Baringo County. Rhipicephalus praetextatus from Ngorongoro crater of Tanzania has been implicated in the transmission of $A$. marginale, the principal agent of bovine anaplasmosis (73). Similarly, we found A. ovis in both Rhipicephalus and Amblyomma ticks in Baringo, but only in Rh. appendiculatus sampled from livestock in Homa Bay County. In contrast, in Baringo County, where no Rh. appendiculatus were sampled, A. ovis occurred most frequently in Am. variegatum. These Anaplasma infections are rarely associated with clinical symptoms in livestock, but may affect livestock health synergistically during coinfections with other livestock disease agents $(74,75)$ in Baringo County.

Sequences of spotted fever group (SFG) Rickettsiae (R. africae, $R$. aeschlimannii, and $R$. rhipicephalii) and three novel Rickettsia spp. were identified in tick ( $R h$. evertsi evertsi and $R h$. pulchellus) samples from livestock and dogs only in Baringo County; no Rickettsia were identified in Homa Bay County, despite the fact that in neighboring Siaya County, a high prevalence of $R$. africae, the etiological agent of African tick bite fever, was previously identified among Am. variegatum parasitizing domestic ruminants (27). In this study, Rickettsia africae was particularly prevalent in Am. gemma (73.68\%) and Am. variegatum (41.02\%) tick pools, but was also found in $R h$. evertsi evertsi $(4.67 \%)$ and 
Rh. pulchellus (5.17\%) tick pools sampled from livestock. We detected $R$. aeschlimannii predominantly in Hyalomma tick pools (Hy. truncatum and Hy. marginatum rufipes) (55.0\%) and incidentally in $R$ h. pulchellus tick pools (3.44\%). In Hy. truncatum tick pools that were sampled from cattle, sheep, and goats, we detected both $A$. bovis and $R$. aeschlimannii. Separate pools of $H y$. marginatum rufipes parasitizing livestock were also positive for $A$. bovis and $R$. aeschlimannii. Rickettsia aeschlimannii has previously been isolated from Hy. truncatum parasitizing camels in the Kano area of Nigeria (76). Though SFG rickettsiosis caused by this TBP has been rarely reported in SSA, it has been reported in humans in Algeria (77) and has also been found in Am. variegatum ticks in western Kenya (27). The likely role of Hyalomma ticks as reservoirs for $R$. aeschlimannii (78) makes these ticks of particular importance in the epidemiology of SFG rickettsiosis in East Africa and warrants further attention. Rickettsia rhipicephalii, as well two of the novel Rickettsia spp. (BR62, BR33), were confined to rhipicephaline (Rh. pulchellus and Rh. eversti evertsi) ticks. A third Rickettsia sp. (TICPA84) was found in Am. gemma (31.57\%) and Rh. evertsi evertsi (4.76\%) tick pools from livestock and dogs. While these findings confirm higher prevalences of Rickettsia spp. in specific Amblyomma and Hyalomma species, their occurrence in Rhipicephalus ticks, perhaps opportunistic, should be considered in the transmission ecology of these TBPs.

From livestock parasitizing ticks in Baringo, we only detected one novel Theileria sp. sequence in $R h$. evertsi evertsi ticks and B. caballi in Rh. pulchellus ticks from cattle, despite the fact that diverse Theileria (79) and Babesia (6) species antigens have been found in livestock surveillance studies in different regions in Kenya. Nonetheless, 29 (0.7\% of sampled ticks) Rh. appendiculatus Neumann 1901, a vector of T. parva (80), were sampled from livestock of Homa Bay County, but not in Baringo. This was contrary to a previous study done over two decades previously, which found $R h$. appendiculatus to be highly prevalent among Zebu cattle grazing along the shores of Lake Victoria in Rusinga Island (25). This change may be an indication of tick control efforts, most likely with acaricides, or difference in sampling period. Although we detected $A$. ovis in three Rh. appendiculatus tick pools, we did not find T. parva, indicating a possible absence of T. parva or extremely low prevalence within our study sites.

Additionally, we found both $H$. fitzsimonsi and Paracoccus sp. bacteria in Am. falsomarmoreum and Am. sparsum ticks from tortoises in Baringo. In South Africa, tortoises have recently been documented to harbor concurrent parasitic infection with H. fitzsimonsi (81). Paracoccus sp. bacteria, also identified in pools of Am. variegatum and Am. gemma ticks sampled from livestock in Baringo County, was first reported in a population of Am. cajennense from South America in 2012, but it is still unknown if Paracoccus infection in ticks is a group of pathogenic rhodobacteraceae or simply plays a role in tick physiology (82). Nonetheless, our findings demonstrate that primers targeting specific Ehrlichia 16S rRNA gene fragment could also be used for detection of Paracoccus species.

While only one to three TBPs were identified in most tick species, none were identified in Ar. persicus sampled from poultry houses, and more than five TBPs were identified in
Rh. evertsi evertsi, Rh. Pulchellus, and Am. gemma tick pools, all of which have been widely implicated in TBP transmission (65, 66, 83-85). Although all 28 pools of Ar. persicus were negative for TBPs, its large population in poultry houses and frequent blood feeding behavior has been linked with nuisance, severe anemia, paralysis, and toxicosis in poultry (86). Furthermore, more TBPs were isolated from Baringo County ticks, suggesting more complex and higher transmission rates in the region, where higher numbers of livestock per household $(>20)$ are kept, compared to the fewer $(<5)$ livestock that are kept by Homa Bay County households.

This study reports the presence, possible circulation, and putative transmission sources of TBPs that are etiological agents of ehrlichiosis, anaplasmosis and rickettsiosis, and hemoparasites of importance to livestock health in the study areas. Among these, we found surprisingly high infection rates of E. ruminantium and E. canis, agents of livestock and canine ehrlichiosis, respectively, in ticks sampled from reptilian hosts, suggesting their likely role as reservoir species in the epidemiology of these TBPs. Proper and rapid diagnoses that include analyses of these TBPs in livestock and their proximal wildlife species are important in mitigating disease burden and possible outbreaks in these areas and the rest of SSA.

\section{ETHICS STATEMENT}

This study was carried out in accordance with the recommendations of the Kenya Medical Research Institute (KEMRI), Wildlife Service (KWS), Directorate of Veterinary Services and Ministry of Health. The protocol was approved by the KEMRI ethics review committee (Approval Ref: non-SSC Protocol \#310) and the KWS Biodiversity Research and Monitoring committee (Permit Ref: KWS/BRM/5001).

\section{AUTHOR CONTRIBUTIONS}

DO, DM, and JV designed the study. DO, EK, and YA conducted the fieldwork. DO, MM, and DOO conducted the laboratory work. DO and JV conducted the analysis and drafted the manuscript; BM, MM, and DM contributed to the interpretation of the data. All the authors contributed to the manuscript editing and approved the final manuscript.

\section{ACKNOWLEDGMENTS}

We acknowledge the technical contribution in sampling of William Owigo and Paul Kitui of icipe's Thomas Odhiambo Campus and Phillip Ojunju of Rusinga Island. We also acknowledge the logistic support of Lillian Igweta, Lisa Omondi, and Margaret Ochanda of icipe's of Capacity Building and Institutional Development unit, and are thankful to Esther Waweru, Caroline Tigoi, and Geoffrey Jagero of icipe's Molecular Biology and Bioinformatics Unit (MBBU) and Martin Lüscher Emerging Infectious Diseases (ML-EID) laboratories. We gratefully acknowledge the contribution of John Tilion of Ruko Community Conservancy Baringo, who facilitated tick collection in the conservancy. 


\section{FUNDING}

This study was supported by the Swedish International Development Cooperation Agency (SIDA), grant number 75000529 to DO as an African Regional Postgraduate Programme in Insect Science (ARPPIS) student. We also acknowledge

\section{REFERENCES}

1. Dunster L, Dunster M, Ofula V, Beti D, Kazooba-Voskamp F, Burt F, et al. First documentation of human Crimean-Congo hemorrhagic fever, Kenya. Emerg Infect Dis (2002) 8:1005-6. doi:10.3201/eid0809.010510

2. Parola P, Paddock CD, Raoult D. Tick-borne rickettsioses around the world: emerging diseases challenging old concepts. Clin Microbiol Rev (2005) 18:719-56. doi:10.1128/CMR.18.4.719-756.2005

3. Parola P. Rickettsioses in sub-Saharan Africa. Ann N Y Acad Sci (2006) 1078:42-7. doi:10.1196/annals.1374.005

4. Sang R, Lutomiah J, Koka H, Makio A, Chepkorir E, Ochieng C, et al. Crimean-Congo hemorrhagic fever virus in Hyalommid ticks, northeastern Kenya. Emerg Infect Dis (2011) 17:1502-5. doi:10.3201/eid1708.102064

5. Lwande OW, Venter M, Lutomiah J, Michuki G, Rumberia C, Gakuya F, et al. Whole genome phylogenetic investigation of a West Nile virus strain isolated from a tick sampled from livestock in north eastern Kenya. Parasit Vectors (2014) 7:542. doi:10.1186/s13071-014-0542-2

6. Kiara H, Jennings A, Bronsvoort B, Handel IG, Mwangi ST, Mbole-Kariuki M, et al. A longitudinal assessment of the serological response to Theileria parva and other tick-borne parasites from birth to one year in a cohort of indigenous calves in Western Kenya. Parasitology (2014) 141:1289-98. doi:10.1017/ S003118201400050X

7. Minakawa N, Sonye G, Dida GO, Futami K, Kaneko S. Recent reduction in the water level of Lake Victoria has created more habitats for Anopheles funestus. Malar J (2008) 7:119. doi:10.1186/1475-2875-7-119

8. Kock RA. What is this infamous "wildlife/livestock disease interface?" A review of current knowledge for the African continent. In: Osofsky SA, editor. Conservation and Development Interventions at the Wildlife/Livestock Interface - Implications for Wildlife, Livestock and Human Health. Switzerland: IUCN (2005). p. 1-13.

9. Dantas-Torres F, Chomel BB, Otranto D. Ticks and tick-borne diseases: a one health perspective. Trends Parasitol (2012) 28:437-46. doi:10.1016/j.pt.2012.07.003

10. Gortazar C, Ferroglio E, Hofle U, Frolich K, Vicente J. Diseases shared between wildlife and livestock: a European perspective. Eur J Wildl Res (2007) 53:241-56. doi:10.1007/s10344-007-0098-y

11. Uilenberg G. International collaborative research: significance of tick-borne hemoparasitic diseases to world animal health. Vet Parasitol (1995) 57:19-41. doi:10.1016/0304-4017(94)03107-8

12. Bengis R, Kock R, Fischer J. Infectious animal diseases: the wildlife/livestock interface. Rev Sci Tech (2002) 21:53-66. doi:10.20506/rst.21.1.1322

13. Olwoch JM, Reyers B, Engelbrecht FA, Erasmus BFN. Climate change and the tick-borne disease, Theileriosis (East Coast fever) in sub-Saharan Africa. J Arid Environ (2008) 72:108-20. doi:10.1016/j.jaridenv.2007.04.003

14. Burridge MJ, Simmons LA, Simbi BH, Peter TF, Mahan SM. Evidence of Cowdria ruminantium infection (heartwater) in Amblyomma sparsum ticks found on tortoises imported into Florida. J Parasitol (2000) 86:1135-6. doi: $10.2307 / 3284836$

15. De Sousa R, Lopes de Carvalho I, Santos AS, Bernardes C, Milhano N, Jesus J, et al. Role of the lizard Teira dugesii as a potential host for Ixodes ricinus tickborne pathogens. Appl Environ Microbiol (2012) 78(10):3767-9. doi:10.1128/ AEM.07945-11

16. Whiley H, Custance G, Graves S, Stenos J, Taylor M, Ross K, et al. Rickettsia detected in the reptile tick Bothriocroton hydrosauri from the lizard Tiliqua rugosa in South Australia. Pathogens (2016) 5(2):E41. doi:10.3390/ pathogens5020041

17. Tijsse-Klassen E, Fonville M, Reimerink JH, Spitzen-van der Sluijs A, Sprong H. Role of sand lizards in the ecology of Lyme and other tick-borne diseases in the Netherlands. Parasit Vectors (2010) 3:42. doi:10.1186/1756-3305-3-42 funding from UK's Department for International Development (DFID), the Swiss Agency for Development and Cooperation (SDC), and the Kenyan Government. The funding bodies did not play a role in the design of this study, the collection, analyses and interpretation of data, the writing of the manuscript, or decision to submit the manuscript for publication.
18. Andoh M, Sakata A, Takano A, Kawabata H, Fujita H, Une Y, et al. Detection of Rickettsia and Ehrlichia spp. in ticks associated with exotic reptiles and amphibians imported into Japan. PLoS One (2015) 10(7):e0133700. doi:10.1371/journal.pone. 0133700

19. Miller RS, Farnsworth ML, Malmberg JL. Diseases at the livestock-wildlife interface: status, challenges, and opportunities in the United States. Prev Vet Med (2013) 110:119-32. doi:10.1016/j.prevetmed.2012.11.021

20. Wiethoelter AK, Beltrán-Alcrudo D, Kock R, Mor SM. Global trends in infectious diseases at the wildlife-livestock interface. Proc Natl Acad Sci U S A (2015) 112:9662-7. doi:10.1073/pnas.1422741112

21. Daszak P, Cunningham AA, Hyatt AD. Anthropogenic environmental change and the emergence of infectious diseases in wildlife. Acta Trop (2001) 78:103-16. doi:10.1016/S0001-706X(00)00179-0

22. Maxwell JF, Lea B-F, Davies TJ. The study of parasite sharing for surveillance of zoonotic diseases. Environ Res Lett (2013) 8:015036. doi:10.1088/1748-9326/8/1/015036

23. Latif AA, Hove T, Kanhai GK, Masaka S. Buffalo-associated Theileria parva: the risk to cattle of buffalo translocation into the Highveld of Zimbabwe. Ann N Y Acad Sci (2002) 969:275-9. doi:10.1111/j.1749-6632.2002.tb04392.x

24. Homewood K, Lewis J. Impact of drought on pastoral livestock in Baringo, Kenya 1983-85. J Appl Ecol (1987) 24:615-31. doi:10.2307/2403897

25. Punyua DK, Latif AA, Nokoe S, Capstick PB. Tick (Acari: Ixodidae) infestations on Zebu cattle in western Kenya: seasonal dynamics of four species of ticks on traditionally managed cattle. J Med Entomol (1991) 28:630-6. doi:10.1093/jmedent/28.5.630

26. Maina AN, Knobel DL, Jiang J, Halliday J, Feikin DR, Cleaveland S, et al. Rickettsia felis infection in febrile patients, Western Kenya, 2007-2010. Emerg Infect Dis (2012) 18:328-31. doi:10.3201/eid1802.111372

27. Maina AN, Jiang J, Omulo SA, Cutler SJ, Ade F, Ogola E, et al. High prevalence of Rickettsia africae variants in Amblyomma variegatum ticks from domestic mammals in rural Western Kenya: implications for human health. Vector Borne Zoonotic Dis (2014) 14:693-702. doi:10.1089/vbz. 2014.1578

28. Njiiri NE, Bronsvoort BMC, Collins NE, Steyn HC, Troskie M, Vorster I, et al. The epidemiology of tick-borne haemoparasites as determined by the reverse line blot hybridization assay in an intensively studied cohort of calves in western Kenya. Vet Parasitol (2015) 210:69-76. doi:10.1016/j.vetpar.2015. 02.020

29. Rubaire-Akiiki C, Okello-Onen J, Nasinyama G, Vaarst M, Kabagambe EK, Mwayi W, et al. The prevalence of serum antibodies to tick-borne infections in Mbale District, Uganda: the effect of agro-ecological zone, grazing management and age of cattle. J Insect Sci (2004) 4:8. doi:10.1673/031.004.0801

30. Oura CA, Tait A, Asiimwe B, Lubega GW, Weir W. Theileria parva genetic diversity and haemoparasite prevalence in cattle and wildlife in and around Lake Mburo National Park in Uganda. Parasitol Res (2011) 108(6):1365-74. doi:10.1007/s00436-010-2030-8

31. Ghai RR, Mutinda M, Ezenwa VO. Limited sharing of tick-borne hemoparasites between sympatric wild and domestic ungulates. Vet Parasitol (2016) 226:167-73. doi:10.1016/j.vetpar.2016.07.005

32. Mwamuye MM, Kariuki E, Omondi D, Kabii J, Odongo D, Masiga D, et al. Novel Rickettsia and emergent tick-borne pathogens: a molecular survey of ticks and tick-borne pathogens in Shimba Hills National Reserve, Kenya. Ticks Tick Borne Dis (2017) 8:208-18. doi:10.1016/j.ttbdis.2016.09.002

33. de la Fuente J, Kocan KM, Almazan C, Blouin EF. Targeting the tick-pathogen interface for novel control strategies. Front Biosci (2008) 13:6947-56. doi: $10.2741 / 3201$

34. Crump JA, Morrissey AB, Nicholson WL, Massung RF, Stoddard RA, Galloway RL, et al. Etiology of severe non-malaria febrile illness in Northern Tanzania: 
a prospective cohort study. PLoS Negl Trop Dis (2013) 7:e2324. doi:10.1371/ journal.pntd.0002324

35. Tigoi C, Lwande O, Orindi B, Irura Z, Ongus J, Sang R. Seroepidemiology of selected arboviruses in febrile patients visiting selected health facilities in the lake/river basin areas of Lake Baringo, Lake Naivasha, and Tana River, Kenya. Vector Borne Zoonotic Dis (2015) 15:124-32. doi:10.1089/vbz.2014.1686

36. Kipkorir EC. Analysis of rainfall climate on the Njemps Flats, Baringo District, Kenya. J Arid Environ (2002) 50:445-58. doi:10.1006/jare.2001.0917

37. Mati BM. The influence of climate change on maize production in the semi-humid-semi-arid areas of Kenya. J Arid Environ (2000) 46:333-44. doi:10.1006/jare.2000.0699

38. Omondi D, Masiga DK, Ajamma YU, Fielding BC, Njoroge L, Villinger J. Unraveling host-vector-arbovirus interactions by two-gene high resolution melting mosquito bloodmeal analysis in a Kenyan wildlife-livestock interface. PLoS One (2015) 10(7):e0134375. doi:10.1371/journal.pone.0134375

39. Onchuru TO, Ajamma YU, Burugu M, Kaltenpoth M, Masiga D, Villinger J. Chemical parameters and bacterial communities associated with larval habitats of Anopheles, Culex and Aedes mosquitoes (Diptera: Culicidae) in Western Kenya. Int J Trop Insect Sci (2016) 36(3):146-60. doi:10.1017/ S1742758416000096

40. Ajamma YU, Villinger J, Omondi D, Salifu D, Onchuru TO, Njoroge L, et al. Composition and genetic diversity of mosquitoes (Diptera: Culicidae) on islands and mainland shores of Kenya's Lakes Victoria and Baringo. J Med Entomol (2016) 53(6):1348-63. doi:10.1093/jme/tjw102

41. Sang R, Kioko E, Lutomiah J, Warigia M, Ochieng C, O'Guinn M, et al. Rift Valley fever virus epidemic in Kenya, 2006/2007: the entomologic investigations. Am J Trop Med Hyg (2010) 83:28-37. doi:10.4269/ajtmh.2010.09-0319

42. Morzaria SP, Musoke AJ, Latif AA. Recognition of Theileria parva antigens by field sera from Rusinga Island, Kenya. Kenya Vet (1988) 12:8.

43. Latif AA, Rowlands GJ, Punyua DK, Hassan SM, Capstick PB. An epidemiological study of tick-borne diseases and their effects on productivity of zebu cattle under traditional management on Rusinga Island, Western Kenya. Prev Vet Med (1995) 22:169-81. doi:10.1016/0167-5877(94)00408-B

44. Walker JB, Keirans JE, Horak IG. The Genus Rhipicephalus (Acari, Ixodidae): A Guide to the Brown Ticks of the World. Cambridge: Cambridge University Press (2000).

45. Walker JB, Olwage A. The tick vectors of Cowdria ruminatum (Ixodoidea: Ixodidae, genus Amblyomma) and their distribution. Onderstepoort J Vet Res (1987) 54(3):353-79.

46. Walker JB. The Ixodid Ticks of Kenya: A Review of Present Knowledge of Their Hosts and Distribution. London: Commonwealth Institute of Entomology (1974).

47. Walker AR, Bouattour A, Camicas J-L, Estrada-Peña A, Horak IG, Latif AA, et al. Ticks of Domestic Animals in Africa: A Guide to Identification of Species. Edinburgh, Scotland: Bioscience Reports (2003).

48. Crowder CD, Rounds MA, Phillipson CA, Picuri JM, Matthews HE, Halverson J, et al. Extraction of total nucleic acids from ticks for the detection of bacterial and viral pathogens. J Med Entomol (2010) 47:89-94. doi:10.1093/ jmedent/47.1.89

49. Tokarz R, Kapoor V, Samuel JE, Bouyer DH, Briese T, Lipkin WI. Detection of tick-borne pathogens by MassTag polymerase chain reaction. Vector Borne Zoonotic Dis (2009) 9:147-52. doi:10.1089/vbz.2008.0088

50. Zhu Y, Fournier PE, Ogata H, Raoult D. Multispacer typing of Rickettsia prowazekii enabling epidemiological studies of epidemic typhus. JClin Microbiol (2005) 43:4708-12. doi:10.1128/JCM.43.9.4708-4712.2005

51. Gubbels JM, de Vos AP, van der Weide M, Viseras J, Schouls LM, de Vries E, et al. Simultaneous detection of Bovine Theileria and Babesia species by reverse line blot hybridization. J Clin Microbiol (1999) 37:1782-9.

52. Katoh K, Standley DM. MAFFT multiple sequence alignment software version 7: improvements in performance and usability. Mol Biol Evol (2013) 30:772-80. doi:10.1093/molbev/mst010

53. Kearse M, Moir R, Wilson A, Stones-Havas S, Cheung M, Sturrock S, et al. Geneious basic: an integrated and extendable desktop software platform for the organization and analysis of sequence data. Bioinformatics (2012) 28(12):1647-9. doi:10.1093/bioinformatics/bts199

54. Altschul SF, Gish W, Miller W, Myers EW, Lipman DJ. Basic local alignment search tool. J Mol Biol (1990) 15(3):403-10. doi:10.1016/ S0022-2836(05)80360-2
55. Saitou N, Nei M. The neighbor-joining method: a new method for reconstructing phylogenetic trees. Mol Biol Evol (1987) 4(4):406-25.

56. Guindon S, Dufayard JF, Lefort V, Anisimova M, Hordijk W, Gascuel O. New algorithms and methods to estimate maximum-likelihood phylogenies: assessing the performance of PHYML 3.0. Syst Biol (2010) 59:307-21. doi:10.1093/sysbio/syq010

57. Drummond AJ, Rambaut A. BEAST: Bayesian evolutionary analysis by sampling trees. BMC Evol Biol (2007) 7:214. doi:10.1186/1471-2148-7-214

58. Hess PN, De Moraes Russo CA. An empirical test of the midpoint rooting method. Biol J Linn Soc (2007) 92:669-74. doi:10.1111/j.1095-8312.2007. 00864.x

59. Collins NE, Liebenberg J, de Villiers EP, Brayton KA, Louw E, Pretorius A. The genome of the heartwater agent Ehrlichia ruminantium contains multiple tandem repeats of actively variable copy number. Proc Natl Acad Sci U S A (2005) 102(3):838-43. doi:10.1073/pnas.0406633102

60. Allsopp M, Visser ES, du Plessis JL, Vogel SW, Allsopp BA. Different organisms associated with heartwater as shown by analysis of $16 \mathrm{~S}$ ribosomal RNA gene sequences. Vet Parasitol (1997) 71(4):283-300. doi:10.1016/ S0304-4017(97)00012-5

61. Wen B, Jian R, Zhang Y, Chen R. Simultaneous detection of Anaplasma marginale and a new Ehrlichia species closely related to Ehrlichia chaffeensis by sequence analyses of $16 \mathrm{~S}$ ribosomal DNA in Boophilus microplus ticks from Tibet. JClin Microbiol (2002) 40(9):3286-90. doi:10.1128/ JCM.40.9.3286-3290.2002

62. Mukhebi AWT, Chamboko CJ, O'Callaghan TF, Peter RL, Kruska GF, Medley SM, et al. An assessment of the economic impact of heartwater (Cowdria ruminantium infection) and its control in Zimbabwe. Prev Vet Med (1999) 39:173-89. doi:10.1016/S0167-5877(98)00143-3

63. Kelly PJ, Lucas H, Yowell C, Beati L, Dame J, Urdaz-Rodriguez J, et al. Ehrlichia ruminantium in Amblyomma variegatum and domestic ruminants in the Caribbean. JMed Entomol (2011) 48:485-8. doi:10.1603/ ME10172

64. Trout Fryxell RT, DeBruyn JM. Correction: the microbiome of Ehrlichiainfected and uninfected lone star ticks (Amblyomma americanum). PLoS One (2016) 11:e0155559. doi:10.1371/journal.pone.0155559

65. Ngumi PN, Rumberia RM, Williamson SM, Sumption KJ, Lesan AC, Kariuki DP. Isolation of the causative agent of heartwater (Cowdria ruminantium) from three Amblyomma species in eight districts of Kenya. Vet Rec (1997) 140:13-6. doi:10.1136/vr.140.1.13

66. Wesonga FD, Mukolwe SW, Rurangirwa F. Cowdria ruminantium identified in Amblyomma gemma using a DNA probe pCS20. Rev Elev Med Vet Pays Trop (1993) 46:179-81.

67. Wesonga FD, Mukolwe SW, Grootenhuis J. Transmission of Cowdria ruminantium by Amblyomma gemma from infected African buffalo (Syncerus caffer) and eland (Taurotragus oryx) to sheep. Trop Anim Health Prod (2001) 33:379-90. doi:10.1023/A:1010539705913

68. Horak IG, McKay IJ, Henen BT, Heyne H, Hofmeyr MD, De Villiers AL. Parasites of domestic and wild animals in South Africa. XLVII. Ticks of tortoises and other reptiles. Onderstepoort J Vet Res (2006) 73:215-27. doi:10.4102/ojvr.v73i3.148

69. Nowak M. The international trade in reptiles (Reptilia) - the cause of the transfer of exotic ticks (Acari: Ixodida) to Poland. Vet Parasitol (2010) 169:373-81. doi:10.1016/j.vetpar.2010.01.006

70. Burgdorfer W, Ormsbee RA, Schmidt ML, Hoogstraal H. A search for the epidemic typhus agent in Ethiopian ticks. Bull World Health Organ (1973) 48:563-9.

71. Wanzala W, Okanga S. Ticks (Acari: Ixodidae) associated with wildlife and vegetation of Haller park along the Kenyan coastline. J Med Entomol (2006) 43:789-94. doi:10.1093/jmedent/43.5.789

72. Kariuki EK, Penzhorn BL, Horak IG. Ticks (Acari: Ixodidae) infesting cattle and African buffaloes in the Tsavo conservation area, Kenya. Onderstepoort $J$ Vet Res (2012) 79:1-4. doi:10.4102/ojvr.v79i1.437

73. Fyumagwa RD, Simmler P, Meli ML, Hoare R, Hofmann-Lehmann R, Lutz $\mathrm{H}$. Prevalence of Anaplasma marginale in different tick species from Ngorongoro Crater, Tanzania. Vet Parasitol (2009) 161:154-7. doi:10.1016/j. vetpar.2008.12.018

74. Hornok S, Edelhofer R, Foldvari G, Joachim A, Farkas R. Serological evidence for Babesia canis infection of horses and an endemic focus of $B$. 
caballi in Hungary. Acta Vet Hung (2007) 55:491-500. doi:10.1556/AVet.55. 2007.4.8

75. Thumbi SM, de C, Bronsvoort BM, Poole EJ, Kiara H, Toye P, et al. Parasite co-infections show synergistic and antagonistic interactions on growth performance of East African zebu cattle under one year. Parasitology (2013) 140:1789-98. doi:10.1017/S0031182013001261

76. Kamani J, Baneth G, Apanaskevich DA, Mumcuoglu KY, Harrus S. Molecular detection of Rickettsia aeschlimannii in Hyalomma spp. ticks from camels (Camelus dromedarius) in Nigeria, West Africa. Med Vet Entomol (2015) 29:205-9. doi:10.1111/mve.12094

77. Mokrani N, Parola P, Tebbal S, Dalichaouche M, Aouati A, Raoult D. Rickettsia aeschlimannii infection, Algeria. Emerg Infect Dis (2008) 14:1814-5. doi:10.3201/eid1411.071221

78. Matsumoto K, Parola P, Brouqui P, Raoult D. Rickettsia aeschlimannii in Hyalomma ticks from Corsica. Eur J Clin Microbiol Infect Dis (2004) 23:732-4. doi:10.1007/s10096-004-1190-9

79. Moumouni PF, Aboge GO, Terkawi MA, Masatani T, Cao S, Kamyingkird $\mathrm{K}$, et al. Molecular detection and characterization of Babesia bovis, Babesia bigemina, Theileria species and Anaplasma marginale isolated from cattle in Kenya. Parasit Vectors (2015) 8:496. doi:10.1186/s13071-015-1106-9

80. Norval RAI, Perry BD, Young A. The Epidemiology of Theileriosis in Africa. London, UK: Academic Press (1992).

81. Cook CA, Smit NJ, Davies AJ. Hemoproteids (Apicomplexa: Haemoproteidae) from South African tortoises (Cryptodira: Testudinidae). J Parasitol (2010) 96:1168-72. doi:10.1645/GE-2527.1

82. Machado-Ferreira E, Piesman J, Zeidner NS, Soares CA. A prevalent alpha-proteobacterium Paracoccus sp. in a population of the cayenne ticks
(Amblyomma cajennense) from Rio de Janeiro, Brazil. Genet Mol Biol (2012) 35:862-7. doi:10.1590/S1415-47572012005000067

83. Mediannikov O, Diatta G, Fenollar F, Sokhna C, Trape JF, Raoult D. Tick-borne rickettsioses, neglected emerging diseases in rural Senegal PLoS Negl Trop Dis (2010) 4(9):e821. doi:10.1371/journal.pntd. 0000821

84. Wanzala W, Ondiaka SN. Tick-borne lymphadenopathy-like condition in an African woman in Kenya. J Res Med Sci (2013) 18:918-21.

85. Kumsa B, Socolovschi C, Almeras L, Raoult D, Parola P. Occurrence and genotyping of Coxiella burnetii in ixodid ticks in Oromia, Ethiopia. Am J Trop Med Hyg (2015) 93:1074-81. doi:10.4269/ajtmh.14-0758

86. Rosenstein M. Paralysis in chickens caused by larvae of the poultry tick, Argas persicus. Avian Dis (1976) 20:407-9. doi:10.2307/1589281

Conflict of Interest Statement: The authors declare that the research was conducted in the absence of any commercial or financial relationships that could be construed as a potential conflict of interest.

Copyright (๑) 2017 Omondi, Masiga, Fielding, Kariuki, Ajamma, Mwamuye, Ouso and Villinger. This is an open-access article distributed under the terms of the Creative Commons Attribution License (CC BY). The use, distribution or reproduction in other forums is permitted, provided the original author(s) or licensor are credited and that the original publication in this journal is cited, in accordance with accepted academic practice. No use, distribution or reproduction is permitted which does not comply with these terms. 